\title{
Synchronization of a Class of Memristive Stochastic Bidirectional Associative Memory Neural Networks with Mixed Time-Varying Delays via Sampled-Data Control
}

\author{
Manman Yuan, ${ }^{1,2}$ Weiping Wang $\mathbb{D},{ }^{1,2}$ Xiong Luo $(\mathbb{D}),{ }^{1,2}$ Chao Ge $\mathbb{D}^{\circ},{ }^{3}$ Lixiang Li $\mathbb{D},{ }^{4}$ \\ Jürgen Kurths, ${ }^{5}$ and Wenbing Zhao $\mathbb{1}^{6}$ \\ ${ }^{1}$ School of Computer and Communication Engineering, University of Science and Technology Beijing, Beijing 100083, China \\ ${ }^{2}$ Beijing Key Laboratory of Knowledge Engineering for Materials Science, Beijing 100083, China \\ ${ }^{3}$ North China University of Science and Technology, Tangshan 063009, China \\ ${ }^{4}$ Information Security Center, State Key Laboratory of Networking and Switching Technology, \\ Beijing University of Posts and Telecommunications, Beijing 100876, China \\ ${ }^{5}$ Potsdam Institute for Climate Impact Research, 14473 Potsdam, Germany \\ ${ }^{6}$ Department of Electrical Engineering and Computer Science, Cleveland State University, Cleveland, OH 44115, USA
}

Correspondence should be addressed to Weiping Wang; shiya666888@126.com and Xiong Luo; xluo@ustb.edu.cn

Received 27 December 2017; Accepted 18 March 2018; Published 30 April 2018

Academic Editor: Jean Jacques Loiseau

Copyright (c) 2018 Manman Yuan et al. This is an open access article distributed under the Creative Commons Attribution License, which permits unrestricted use, distribution, and reproduction in any medium, provided the original work is properly cited.

The paper addresses the issue of synchronization of memristive bidirectional associative memory neural networks (MBAMNNs) with mixed time-varying delays and stochastic perturbation via a sampled-data controller. First, we propose a new model of MBAMNNs with mixed time-varying delays. In the proposed approach, the mixed delays include time-varying distributed delays and discrete delays. Second, we design a new method of sampled-data control for the stochastic MBAMNNs. Traditional control methods lack the capability of reflecting variable synaptic weights. In this paper, the methods are carefully designed to confirm the synchronization processes are suitable for the feather of the memristor. Third, sufficient criteria guaranteeing the synchronization of the systems are derived based on the derive-response concept. Finally, the effectiveness of the proposed mechanism is validated with numerical experiments.

\section{Introduction}

Associate memory is one of the most significant activities of human brain, which can be applied in study of brain-like systems [1], intelligent thinking for intelligent robots [2], and so on. Since Kosko discussed the concept of bidirectional associative memory neural networks (BAMNNs) [3] in 1988, BAMNNs occupied the great researchers' time and have been studied for several years. Nowadays, due to the wide applications in signal processing, associative memory, pattern recognition, and so on [4], chaos control and synchronization of BAMNNs have been intensively investigated. Owing to the special characters of the memristor [5], researchers have replaced resistor with memristor in large scale integration circuits to construct the MBAMNNs [6]. MBAMNNs are more suitable for mimicking the associative memory process of human brain contrast with the BAMNNs. Thus, more and more researchers build the MBAMNNs models for investigating a variety of applications [7-10].

In practical MBAMNNs systems, an ever-present phenomenon is the threshold of the sensitive memristor with a nonlinear drift effect [11], such as the voltages, current, and magnetic flux. It is well known that the presence of the threshold input may drastically deteriorate the desired performance, even inducing the inaccuracy of closed-loop systems under investigation. Hence, it is important to take the nonlinear characteristic of the MBAMNNs into consideration in the dynamical systems. In general, two common 
methods are utilized to cope with the nonlinear characteristic of the MBAMNNs. One is treating the parameters as constants [12, 13], and the other is making a study of antisynchronization [14]. Recently, the problem of nonlinear characteristic has also been considered to the field of memristive neural networks (MNNs) [15-22]. Although the importance of nonlinear characteristic has been rather well recognized, but few related results have been reported on the synchronization of MBAMNNs. This is the first motivation of the present paper.

Synchronization is an elementary collective phenomenon that enables coherent behavior in neural networks (NNs), where neurons coact with each other and achieve a common dynamic behavior. Synchronization is of great significance for its potential applications in many areas, including harmonic oscillation generation, biology systems, and secure communication. Looking through the literatures on the synchronization of MBAMNNs, one can find that most of the results are based on the two kinds of continuous-time control strategies: the state feedback control [23-28] and adaptive control [29]. A prerequisite of these approaches is that the controllers must obtain signals from sensors in a continuous way [30]. This will increase the control cost heavily and cause a waste of communication bandwidth [31].

In contrast to the continuous-time control, the sampleddata control merely makes use of the sampling signals at discrete time instants. Consequently, the sampled-data control can eliminate the continuous monitoring of system states as well as the continuous information transmission. Therefore, the sampled-data control is a more managing choice in applications. Till now, numerous results have been reported in this aspect [30-33]. However, to the best of our knowledge, there are few relevant achievements that consider the sampled-data synchronization of MBAMNNs. This is the second motivation of the present paper.

Owing to the limited speed of signal transmission between the neurons, the finite switching speed between different circuit elements in hardware implementations of NNs, and the viscosity of synapses triggered by biological NNs, time delay is an inevitable phenomenon in NNs. There are many types of delays, like discrete delay, leakage time delay, distributed delay, neutral-type delay, and so on. These delays are the main factors that contribute to the oscillation, instability, and the performance degradation to the dynamical systems [34]. Therefore, the dynamic systems with time delay becomes a hot topic in the theoretical and application realms.

Besides, the actual communication between real systems is usually disturbed by a stochastic perturbation from various uncertainties. In secure communication systems, the digital signal is transmitted by switching forth and back continuously between synchronization. The stochastic perturbation will probably lead to package losses or influences the signal transmission. Hence, it is important to discuss the effect of probabilistic delays, stochastic perturbations, and so on [3538]. Therefore, it is valuable and practical to discuss the effect of the stochastic perturbations and time-varying delays on MBAMNNs.

Motivated by the foregoing discussions, this paper aims at investigating the synchronization of MBAMNNs with mixed time-varying delays and stochastic perturbations by designing a suitable sampled-data controller. The main contributions of this paper are summarized as follows.

(1) We first investigate the globally asymptotic synchronization of MBAMNNs with mixed time-varying delays and stochastic perturbations.

(2) According to the characters of memristor, we consider the parameters mismatch between the drive-response systems and design a suitable sampled-data controller to fit the features of the memristor.

(3) We also analyze the feasible region of the sampling period according to simulations, which is significant to some potential future research. Owing to the sampled-data synchronization analysis, Lyapunov functional method, and stochastic analysis theory, the synchronization criteria of the parameters mismatched MBAMNNs are derived.

The rest of this paper is organized as follows. The systems and problems formulation are presented in Section 2. In Section 3, based on Lyapunov functional method, stochastic analysis theory, and inequality techniques, sufficient criteria that depend on such system for synchronization are obtained. Numerical simulations are demonstrated to verify the effectiveness of the obtained results in Section 4. Finally, conclusions are given in Section 5.

\section{Model Description and Preliminaries}

2.1. Model Description. In order to better understand the MBAMNNs, firstly we describe the circuit of a general class of BAMNNs with the architecture as shown in Figure 1. Take the $i$ th subsystem and the $j$ th subsystem as the unit of analysis so as to simplify illustration [39]; one can clearly see that the Kirchhoff's current law (KCL) of the subsystems of BAMNNs [3] is described as the following differential equation:

$$
\begin{aligned}
& d x_{i}(t)=-x_{i}(t)+\frac{1}{\mathscr{C}_{i}} \sum_{j=1}^{m} \frac{f_{j}\left(y_{j}(t-\tau(t))\right)}{\mathscr{F}_{j i}} \times \operatorname{sign}_{j i} \\
&+\frac{\mathscr{I}_{i}(t)}{\mathscr{C}_{j}}, \quad t \geq 0, i=1,2, \ldots, n, \\
& d y_{j}(t)=-y_{j}(t)+\frac{1}{\widetilde{\mathscr{C}}_{i}} \sum_{i=1}^{n} \frac{g_{i}\left(x_{i}(t-\tau(t))\right)}{\mathscr{R}_{i j}} \times \operatorname{sign}_{i j} \\
&+\frac{\mathscr{J}_{j}(t)}{\widetilde{\mathscr{C}}_{j}}, \quad t \geq 0, j=1,2, \ldots, m,
\end{aligned}
$$

where $x_{i}(t)$ and $y_{j}(t)$ are the voltages of capacitors $\mathscr{C}_{i}$ and $\widetilde{\mathscr{C}}_{j}$, respectively. And $\mathscr{F}_{j i}$ presents the resistor between the feedback function $f_{j}\left(y_{j}(t-\tau(t))\right)$ and $x_{i}(t) ; \mathscr{R}_{i j}$ depicts the resistor between the feedback function $g_{i}\left(x_{i}(t-\tau(t))\right)$ and $y_{j}(t)$. Then the transmission time-varying delay is illustrated by $\tau(t), \mathscr{I}_{i}(t)$ is the bias function or external input on the $i$ th 


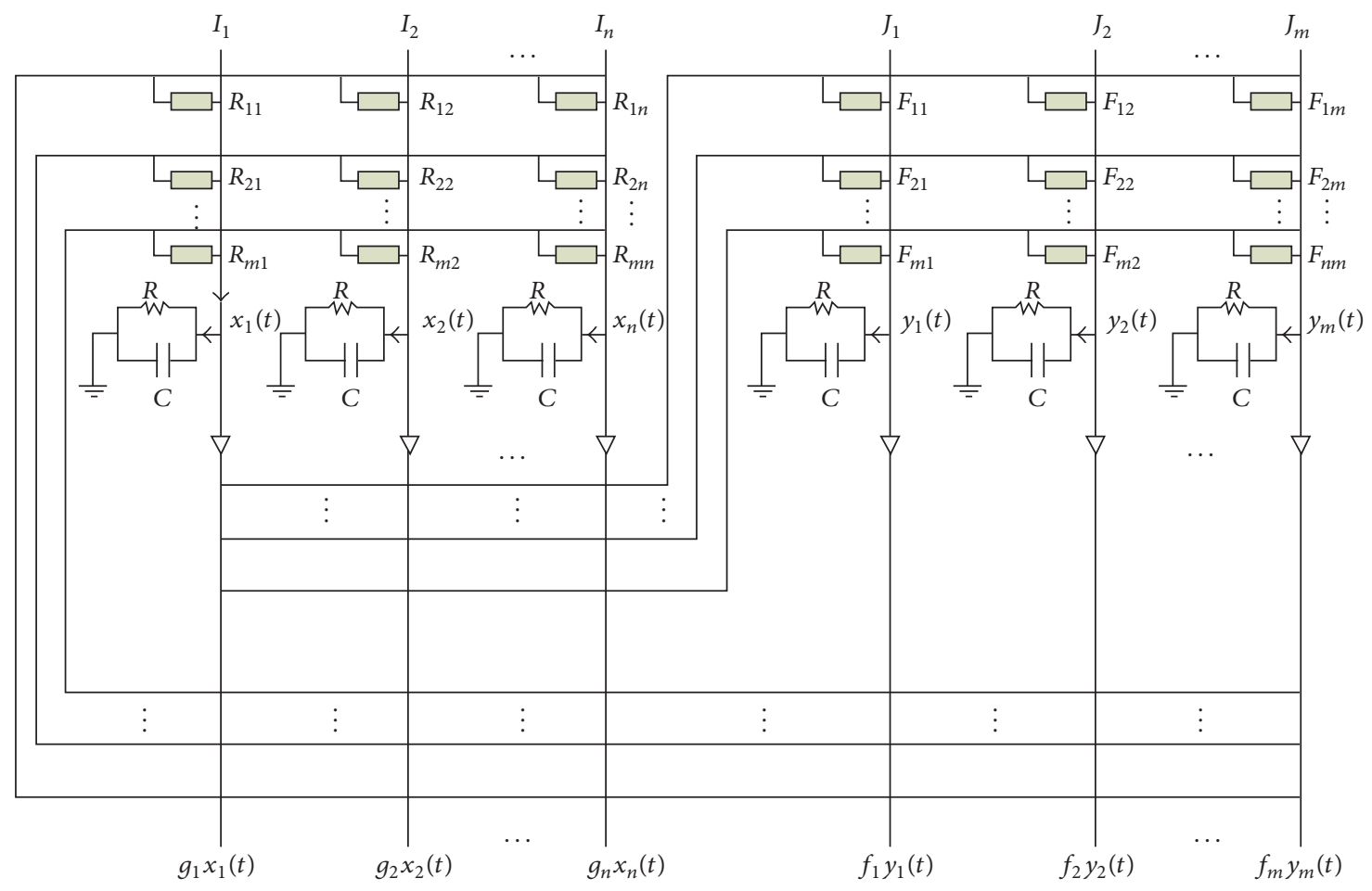

FIgURE 1: The circuits implementing of BAMNNs with transmission time-varying delay.

subsystem at time $t, \mathscr{F}_{j}(t)$ denotes the bias function or external input on the $j$ th subsystem at time $t$, and

$$
\operatorname{sign}_{i j}=\operatorname{sign}_{j i} \begin{cases}1, & i \neq j, \\ -1, & i=j .\end{cases}
$$

Remark 1. Enlightened by [23-29], especially for [39], we proposed the following system which contains not only discrete time-varying delays $\tau(t)$ and $\sigma(t)$, but also distributed time-varying delays $\mu(t)$ and $\varepsilon(t)$. And self-inhibition weights $d_{i}\left(x_{i}(t)\right)$ and $p_{j}\left(y_{j}(t)\right)$ are also time-varying. Therefore, the obtained results are more general and practical than some existing results.

Based on the physical properties of a memristor, the proposed MBAMNNs with mixed time-varying delays are described by the following differential equations:

$$
\begin{aligned}
& d x_{i}(t)=\left[-d_{i}\left(x_{i}(t)\right) x_{i}(t)+I_{i}(t)\right. \\
& +\sum_{j=1}^{m} a_{j i}\left(x_{i}(t)\right) f_{j}\left(y_{j}(t)\right) \\
& +\sum_{j=1}^{m} b_{j i}\left(x_{i}(t-\tau(t))\right) f_{j}\left(y_{j}(t-\tau(t))\right)
\end{aligned}
$$

$$
\begin{aligned}
& \left.+\sum_{j=1}^{m} c_{j i}\left(x_{i}(t)\right) \int_{t-\mu(t)}^{t} f_{j}\left(y_{j}(s)\right) d s\right] d t+\sum_{j=1}^{m} \beta_{j i} \\
& \cdot\left(t, y_{j}(t), y_{j}(t-\tau(t))\right) d \omega_{j}(t), \\
& d y_{j}(t)=\left[-p_{j}\left(y_{j}(t)\right) y_{j}(t)+I_{j}(t)\right. \\
& +\sum_{i=1}^{n} m_{i j}\left(y_{j}(t)\right) g_{i}\left(x_{i}(t)\right) \\
& +\sum_{i=1}^{n} n_{i j}\left(y_{j}(t-\sigma(t))\right) g_{i}\left(x_{i}(t-\sigma(t))\right) \\
& \left.+\sum_{i=1}^{n} q_{i j}\left(y_{j}(t)\right) \int_{t-\varepsilon(t)}^{t} g_{i}\left(x_{i}(s)\right) d s\right] d t+\sum_{i=1}^{n} \beta_{i j} \\
& \cdot\left(t, x_{i}(t), x_{i}(t-\sigma(t))\right) d \omega_{i}(t),
\end{aligned}
$$

where $x_{i}(t)$ and $y_{j}(t)$ denote the voltages of capacitors $C_{i}$ and $\widetilde{C}_{j}$ at time $t$, for $t \geq 0, i=1,2, \ldots, n$, and $j=1,2, \ldots, m . d_{i}>$ 0 and $p_{j}>0$ represent the self-feedback connection weight. Then $a_{j i}\left(x_{i}(t)\right), b_{j i}\left(x_{i}(t-\tau(t))\right), c_{j i}\left(x_{i}(t)\right), m_{i j}\left(y_{j}(t)\right), n_{i j}\left(y_{j}(t-\right.$ $\sigma(t)))$, and $q_{i j}\left(y_{j}(t)\right)$ represent the memristor-based weights. In addition, $f_{j}\left(y_{j}(t)\right)$ and $g_{i}\left(x_{i}(t)\right)$ are feedback functions, $\tau(t)$ and $\sigma(t)$ are discrete time-varying delays, $\mu(t)$ and $\varepsilon(t)$ are finite distributed time-varying delays. In addition, $I_{i}(t)$ and $I_{j}(t)$ denote the continuous external inputs, respectively. 
According to the current-voltage characteristic and the property of a memristor, the memristive connection weights of system (3) can be modeled as

$$
\begin{aligned}
d_{i}\left(x_{i}(t)\right) & = \begin{cases}\check{d}_{i}, & \left|x_{i}(t)\right| \leq T_{i}, \\
\widehat{d}_{i}, & \left|x_{i}(t)\right|>T_{i},\end{cases} \\
a_{j i}\left(x_{i}(t)\right) & = \begin{cases}\check{a}_{j i}, & \left|x_{i}(t)\right| \leq T_{i}, \\
\widehat{a}_{j i}, & \left|x_{i}(t)\right|>T_{i},\end{cases} \\
p_{j}\left(y_{j}(t)\right) & = \begin{cases}\check{p}_{j}, & \left|y_{j}(t)\right| \leq R_{j}, \\
\widehat{p}_{j}, & \left|y_{j}(t)\right|>R_{j},\end{cases} \\
m_{i j}\left(y_{j}(t)\right) & = \begin{cases}\check{m}_{i j}, & \left|y_{j}(t)\right| \leq R_{j}, \\
\widehat{m}_{i j}, & \left|y_{j}(t)\right|>R_{j},\end{cases} \\
c_{j i}\left(x_{i}(t)\right) & = \begin{cases}\check{c}_{j i}, & \left|x_{i}(t)\right| \leq T_{i}, \\
\widehat{c}_{j i}, & \left|x_{i}(t)\right|>T_{i},\end{cases} \\
q_{j i}\left(x_{j}(t)\right) & = \begin{cases}\check{q}_{i j}, & \left|y_{j}(t)\right| \leq R_{j}, \\
\widehat{q}_{i j}, & \left|y_{j}(t)\right|>R_{j},\end{cases} \\
n_{i j}\left(y_{j}(t-\sigma(t))\right) & = \begin{cases}\check{b}_{j i}, & \left|x_{i}(t-\tau(t))\right| \leq T_{i}, \\
\widehat{b}_{j i}, & \left|x_{i}(t-\tau(t))\right|>T_{i}, \\
\check{n}_{i j}, & \left|y_{j}(t-\sigma(t))\right| \leq x_{j}(t-\sigma(t)) \mid>R_{j},\end{cases}
\end{aligned}
$$

in which switching jumps $T_{i}>0, \check{d}_{i}>0, \widehat{d}_{i}>0$ and $\check{a}_{j i}, \widehat{a}_{j i}$, $\check{b}_{j i}, \widehat{b}_{j i}, \check{c}_{j i}, \widehat{c}_{j i}$, for $i=1,2, \ldots, n, j=1,2, \ldots, m$ are constants, so do $R_{j}, \check{p}_{j}, \widehat{p}_{j}, \check{m}_{i j}, \widehat{m}_{i j}, \check{q}_{i j}, \widehat{q}_{i j}, \check{n}_{i j}$, and $\widehat{n}_{i j}$.

In this paper, we treat system (3) as the drive system; then the corresponding response system with stochastic perturbations is described as

$$
\begin{aligned}
& d \widehat{x}_{i}(t)=\left[-d_{i}\left(\widehat{x}_{i}(t)\right) \hat{x}_{i}(t)+\sum_{j=1}^{m} a_{j i}\left(\widehat{x}_{i}(t)\right) f_{j}\left(\hat{y}_{j}(t)\right)\right. \\
& +\sum_{j=1}^{m} b_{j i}\left(\widehat{x}_{i}(t-\tau(t))\right) f_{j}\left(\hat{y}_{j}(t-\tau(t))\right) \\
& +\sum_{j=1}^{m} c_{j i}\left(\hat{x}_{i}(t)\right) \int_{t-\mu(t)}^{t} f_{j}\left(\hat{y}_{j}(s)\right) d s+I_{i}(t) \\
& \left.+U_{i}(t)\right] d t+\sum_{j=1}^{m} \beta_{j i} \\
& \cdot\left(t, \hat{y}_{j}(t), \hat{y}_{j}(t-\tau(t))\right) d \omega_{j}(t)
\end{aligned}
$$

$$
\begin{aligned}
& d \widehat{y}_{j}(t)=\left[-p_{j}\left(\widehat{y}_{j}(t)\right) \hat{y}_{j}(t)\right. \\
& +\sum_{i=1}^{n} m_{i j}\left(\hat{y}_{j}(t)\right) g_{i}\left(\widehat{x}_{i}(t)\right) \\
& +\sum_{i=1}^{n} n_{i j}\left(\widehat{y}_{j}(t-\sigma(t))\right) g_{i}\left(\widehat{x}_{i}(t-\sigma(t))\right) \\
& +\sum_{i=1}^{n} q_{i j}\left(\hat{y}_{j}(t)\right) \int_{t-\varepsilon(t)}^{t} g_{i}\left(\widehat{x}_{i}(s)\right) d s+I_{j}(t) \\
& \left.+U_{j}(t)\right] d t+\sum_{i=1}^{n} \beta_{i j} \\
& \quad \cdot\left(t, \widehat{x}_{i}(t), \widehat{x}_{i}(t-\sigma(t))\right) d \omega_{i}(t)
\end{aligned}
$$

where

$$
\begin{aligned}
& d_{i}\left(\widehat{x}_{i}(t)\right)= \begin{cases}\check{d}_{i}, & \left|\widehat{x}_{i}(t)\right| \leq T_{i}, \\
\widehat{d}_{i}, & \left|\widehat{x}_{i}(t)\right|>T_{i},\end{cases} \\
& a_{j i}\left(\widehat{x}_{i}(t)\right)= \begin{cases}\check{a}_{j i}, & \left|\widehat{x}_{i}(t)\right| \leq T_{i}, \\
\widehat{a}_{j i}, & \left|\widehat{x}_{i}(t)\right|>T_{i},\end{cases} \\
& c_{j i}\left(\widehat{x}_{i}(t)\right)= \begin{cases}\check{c}_{j i}, & \left|\widehat{x}_{i}(t)\right| \leq T_{i}, \\
\widehat{c}_{j i}, & \left|\widehat{x}_{i}(t)\right|>T_{i},\end{cases} \\
& p_{j}\left(\widehat{y}_{j}(t)\right)= \begin{cases}\check{p}_{j}, & \left|\hat{y}_{j}(t)\right| \leq R_{j}, \\
\widehat{p}_{j}, & \left|\hat{y}_{j}(t)\right|>R_{j},\end{cases} \\
& m_{i j}\left(\widehat{y}_{j}(t)\right)= \begin{cases}\check{m}_{i j}, & \left|\hat{y}_{j}(t)\right| \leq R_{j}, \\
\widehat{m}_{i j}, & \left|\hat{y}_{j}(t)\right|>R_{j},\end{cases} \\
& q_{i j}\left(\hat{y}_{j}(t)\right)= \begin{cases}\check{q}_{i j}, & \left|\hat{y}_{j}(t)\right| \leq R_{j}, \\
\widehat{q}_{i j}, & \left|\hat{y}_{j}(t)\right|>R_{j},\end{cases} \\
& b_{j i}\left(\widehat{x}_{i}(t-\tau(t))\right)= \begin{cases}\check{b}_{j i}, & \left|\widehat{x}_{i}(t-\tau(t))\right| \leq T_{i}, \\
\widehat{b}_{j i}, & \left|\widehat{x}_{i}(t-\tau(t))\right|>T_{i},\end{cases} \\
& n_{i j}\left(\hat{y}_{j}(t-\sigma(t))\right)= \begin{cases}\check{n}_{i j}, & \left|\widehat{y}_{j}(t-\sigma(t))\right| \leq R_{j}, \\
\widehat{n}_{i j}, & \left|\hat{y}_{j}(t-\sigma(t))\right|>R_{j} .\end{cases}
\end{aligned}
$$

$U_{i}(t)$ and $U_{j}(t)$ are the appropriate controllers that will be proposed in order to gain the certain control objectives. Consider the following state feedback sampled-data controllers:

$$
\begin{aligned}
& U_{i}(t)=K_{i} e_{i}\left(t_{k 1}\right)-\lambda_{i} \operatorname{sign}\left(e_{i}(t)\right), \\
& U_{j}(t)=K_{j} e_{j}\left(t_{k 2}\right)-\lambda_{j} \operatorname{sign}\left(e_{j}(t)\right),
\end{aligned}
$$

where $K_{i}$ and $K_{j}$ are the sampled-data controllers gain matrices to be designed. $\lambda_{i}$ and $\lambda_{j}$ are positive scalars. Then $e_{i}\left(t_{k 1}\right)$ 
and $e_{j}\left(t_{k 2}\right)$ are discrete measurements of $e_{i}(t)$ and $e_{j}(t)$ at the sampling instant $t_{k 1}$ and $t_{k 2}$, respectively. And the sampling instants satisfy the following conditions:

$$
\begin{aligned}
& 0=t_{01}<t_{11}<\cdots<t_{k 1}<\cdots<\lim _{n \rightarrow \infty} t_{k 1}=+\infty, \\
& 0=t_{02}<t_{12}<\cdots<t_{k 2}<\cdots<\lim _{n \rightarrow \infty} t_{k 2}=+\infty .
\end{aligned}
$$

Remark 2. According to the discussions above, the inner connection matrices $d_{i}\left(x_{i}(t)\right), a_{j i}\left(x_{i}(t), b_{j i}\left(x_{i}(t-\tau(t))\right), c_{j i}\left(x_{i}(t)\right)\right.$, $p_{j}\left(y_{j}(t)\right), m_{i j}\left(y_{j}(t)\right), q_{i j}\left(y_{j}(t)\right), n_{i j}\left(y_{j}(t-\sigma(t))\right), d_{i}\left(\widehat{x}_{i}(t)\right)$, $a_{j i}\left(\widehat{x}_{i}(t), b_{j i}\left(\hat{x}_{i}(t-\tau(t))\right), c_{j i}\left(\widehat{x}_{i}(t)\right), p_{j}\left(\widehat{y}_{j}(t)\right), m_{i j}\left(\hat{y}_{j}(t)\right)\right.$, $q_{i j}\left(\hat{y}_{j}(t)\right)$, and $n_{i j}\left(\hat{y}_{j}(t-\sigma(t))\right)$ of systems (3) and (5) are varying with the state of memristance. Therefore, the MBAMNNs are considered as the state-dependent systems. When the parameters are all constants, systems (3) and (5) become a general class of BAMNNs.

We define the following error system as follows:

$$
\begin{aligned}
& d e_{i}(t)=d \widehat{x}_{i}(t)-d x_{i}(t), \quad i=1,2, \ldots, n, \\
& d e_{j}(t)=d \widehat{y}_{j}(t)-d y_{j}(t), \quad j=1,2, \ldots, m .
\end{aligned}
$$

Remark 3. Some of the published papers studied the synchronization of MNNs $[24,25]$ through the following assumption to design the error system:

$$
\begin{gathered}
\operatorname{co}\left[\underline{a}_{i j}, \bar{a}_{i j}\right] f_{j}\left(y_{j}(t)\right)-\operatorname{co}\left[\underline{a}_{i j}, \bar{a}_{i j}\right] f_{j}\left(x_{j}(t)\right) \\
\subseteq \operatorname{co}\left[\underline{a}_{i j}, \bar{a}_{i j}\right]\left(f_{j}\left(y_{j}(t)\right)-f_{j}\left(x_{j}(t)\right)\right) .
\end{gathered}
$$

Let $\operatorname{co}[a, b]$ denote the closure of convex hull generated by real numbers $a$ and $b$ or real matrices $a$ and $b$. However this assumption has not always been proved to be correct. In [26], authors tried to deal with the synchronization issue of MABMNNs, but the results are unreasonable without taking the switching jumps into account.

The antisynchronization can avoid the problems mentioned above by constructing the error system as the following assumption:

$$
\begin{gathered}
\operatorname{co}\left[\underline{a}_{i j}, \bar{a}_{i j}\right] f_{j}\left(y_{j}(t)\right)+\operatorname{co}\left[\underline{a}_{i j}, \bar{a}_{i j}\right] f_{j}\left(x_{j}(t)\right) \\
\subseteq \operatorname{co}\left[\underline{a}_{i j}, \bar{a}_{i j}\right]\left(f_{j}\left(y_{j}(t)\right)+f_{j}\left(x_{j}(t)\right)\right) .
\end{gathered}
$$

But it still has limitations in practical applications, such as associative memory and associative learning. It is worth mentioning that we fully consider the complex property of the inner connection and take the switching jumps into consideration. Therefore, the obtained results are more practical and less conservative than some published literatures.

2.2. Definitions and Assumptions. In order to get our primary conclusions in the next section, we make the following assumptions. For the sampling interval, one has the following.

Assumption 4 (see [40]). It is supposed that the interval between any two sampling instants is bounded by $d(d>0)$ :

$$
\begin{aligned}
& \Delta_{k 1}=t_{k 1+1}-t_{k 1} \leq d_{1}, \quad \forall k_{1} \geq 0, \\
& \Delta_{k 2}=t_{k 2+1}-t_{k 2} \leq d_{2}, \quad \forall k_{2} \geq 0 .
\end{aligned}
$$

The constants $d_{1}, d_{2}$ denote the maximum time span between $t_{k 1+1}$ and $t_{k 1}, t_{k 2+1}$ and $t_{k 2}$. The state is sampled, and $t_{k 1+1}, t_{k 2+1}$ are the next update reaching the destination.

Due to the discrete terms $e_{i}\left(t_{k 1}\right)$ and $e_{j}\left(t_{k 2}\right)$, it is difficult to analyze the synchronization of MBAMNNs directly. Therefore, according to the input delay approach, $t_{k 1}$ and $t_{k 2}$ are defined as

$$
\begin{aligned}
& t_{k 1}=t-\left(t-t_{k 1}\right):=t-d_{1}(t), \\
& t_{k 2}=t-\left(t-t_{k 2}\right):=t-d_{2}(t),
\end{aligned}
$$

where $0 \leq d_{1}(t) \leq d_{1}, 0 \leq d_{2}(t) \leq d_{2}$, and the controllers can be designed as

$$
\begin{aligned}
& U_{i}(t)=K_{i} e_{i}\left(t-d_{1}(t)\right)-\lambda_{i} \operatorname{sign}\left(e_{i}(t)\right), \\
& U_{j}(t)=K_{j} e_{j}\left(t-d_{2}(t)\right)-\lambda_{j} \operatorname{sign}\left(e_{j}(t)\right) .
\end{aligned}
$$

Remark 5. It should be noted that [41] has made use of the sampled-data control to the MNNs, but to the best of our knowledge little attention has been paid to the MBAMNNs with mixed time-varying delays and stochastic perturbations based on sampled-data control theory, which motivates our present study.

Definition 6. Systems (3) and (5) are said to be asymptotically synchronized if and only if the error systems are globally asymptotically stable for the equilibrium points $e_{i}(t) \equiv 0$ and $e_{j}(t) \equiv 0$. That is, $e_{i}(t) \rightarrow 0, e_{j}(t) \rightarrow 0$ as $t \rightarrow 0$, for any initial conditions

$$
\begin{aligned}
\phi(s) & =\left(\phi_{1}(s), \phi_{2}(s), \ldots, \phi_{n}(s)\right)^{T} \in \mathscr{C}\left([-v, 0], \mathbb{R}^{n}\right), \\
\gamma(s) & =\left(\gamma_{1}(s), \gamma_{2}(s), \ldots, \gamma_{m}(s)\right)^{T} \in \mathscr{C}\left([-\varsigma, 0], \mathbb{R}^{m}\right), \\
\varphi(s) & =\left(\varphi_{1}(s), \varphi_{2}(s), \ldots, \varphi_{n}(s)\right)^{T} \in \mathscr{C}\left([-\nu, 0], \mathbb{R}^{n}\right), \\
\psi(s) & =\left(\psi_{1}(s), \psi_{2}(s), \ldots, \psi_{m}(s)\right)^{T} \\
& \in \mathscr{C}\left([-\varsigma, 0], \mathbb{R}^{m}\right),
\end{aligned}
$$

where $\nu=\max _{1 \leq i \leq n}\left\{\tau_{i}, \mu_{i}\right\}$ which denotes the Banach space of all continuous functions mapping $[-\nu, 0]$ into $\mathbb{R}^{n}$ with 2norm defined by $\|\phi\|=\left(\sum_{i=1}^{n} \phi_{i}^{2}\right),\|\varphi\|=\left(\sum_{i=1}^{n} \varphi_{i}^{2}\right)$, and $\varsigma=\max _{1 \leq j \leq m}\left\{\sigma_{j}, \varepsilon_{j}\right\}$. They denote the Banach space of all continuous functions mapping $[-\varsigma, 0]$ into $\mathbb{R}^{m}$ with 2 -norm defined by $\|\gamma\|=\left(\sum_{j=1}^{m} \gamma_{j}^{2}\right),\|\psi\|=\left(\sum_{j=1}^{m} \psi_{j}^{2}\right)$.

Assumption 7 (see [42]). The activation functions $f_{j}(\cdot)(j=$ $1,2, \ldots, m)$ and $g_{i}(\cdot)(i=1,2, \ldots, n)$ are bounded and globally Lipschitz continuous in $\mathbb{R}$; namely, there exist constants $\alpha_{i}, \beta_{i}, \alpha_{j}$, and $\beta_{j}$ for all $s_{1}, s_{2} \in \mathbb{R}, s_{1} \neq s_{2}$ such that

$$
\begin{array}{ll}
\alpha_{j} \leq \frac{f_{j}\left(s_{1}\right)-f_{j}\left(s_{2}\right)}{s_{1}-s_{2}} \leq \beta_{j}, & \left|f_{j}(\cdot)\right| \leq \Gamma_{j}, \\
\alpha_{i} \leq \frac{g_{i}\left(s_{1}\right)-g_{i}\left(s_{2}\right)}{s_{1}-s_{2}} \leq \beta_{i}, & \left|g_{i}(\cdot)\right| \leq \Delta_{i},
\end{array}
$$

where the constants $\alpha_{i}, \beta_{i}, \alpha_{j}$, and $\beta_{j}$ can be positive numbers, negative numbers, or zero. 
Assumption 8 (see [37]). There exist constants $R_{1} \geqslant 0, R_{2} \geqslant 0$, such that

$$
\begin{aligned}
& \operatorname{Trace}\left[\sigma^{T}(t, x(t), x(t-\xi(t)))\right. \\
& \quad \cdot \sigma(t, x(t), x(t-\xi(t)))] \leq x^{T}(t) R_{1} x(t)+x^{T}(t \\
& \quad-\xi(t)) R_{2} x(t-\xi(t)) .
\end{aligned}
$$

For the stochastic system [42],

$$
d y(t)=g(t, y(t)) d t+\sigma(t, y(t)) d \omega(t)
$$

where $\omega(t)$ is the Brownian motion and it is clearly $\mathbb{E} \omega(t)=0$. $\mathscr{L}$ is the operator defined as follows:

$$
\begin{aligned}
\mathscr{L} V(t, y)= & V_{t}(t, y)+V_{y}(t, y) \\
& +\frac{1}{2} \operatorname{Trace}\left[\sigma^{T}(t, y(t)) V_{y y} \sigma(t, y(t))\right],
\end{aligned}
$$

where

$$
\begin{aligned}
V_{t}(t, y) & =\frac{\partial V_{t}(t, y)}{\partial t}, \\
V_{y y}(t, y) & =\left(\frac{\partial^{2} V_{t}(t, y)}{\partial y_{i} \partial y_{j}}\right), \\
V_{y}(t, y) & =\left(\frac{\partial V_{t}(t, y)}{\partial y_{1}}, \frac{\partial V_{t}(t, y)}{\partial y_{2}}, \ldots, \frac{\partial V_{t}(t, y)}{\partial y_{n}}\right)^{T} .
\end{aligned}
$$

Assumption 9. The time-varying delays $\tau(t), \sigma(t)$ in this paper are differential functions, where

$$
\begin{aligned}
0 & <\tau(t)<\tau, \\
0 & <\sigma(t)<\sigma, \\
\dot{\tau}(t) & \leq \tau_{1}<1, \\
\dot{\sigma}(t) & \leq \sigma_{1}<1, \\
\mu(t) & \leq \mu, \\
\varepsilon & \leq \varepsilon, \\
\dot{d}_{1}(t) & \leq d_{1}^{\prime}<1, \\
\dot{d}_{2}(t) & \leq d_{2}^{\prime}<1,
\end{aligned}
$$

for all $t \geq 0$.

Lemma 10 (see [43]). Given any real matrix $X, Z, P$ of appropriate dimensions, a scalar $\epsilon_{0}>0$, and $P>0$, the following inequality holds:

$$
X^{T} Z+Z^{T} X \leq \epsilon_{0} X^{T} P X+\epsilon_{0}^{-1} Z^{T} P^{-1} Z
$$

In particular, if $X$ and $Z$ are vectors, $X^{T} Z \leq(1 / 2)\left(X^{T} X+\right.$ $\left.Z^{T} Z\right)$

\section{Main Results}

In this section, we get some new sufficient conditions to ensure the synchronization of MBAMNNs by the designed sampled-data controller.

Theorem 11. Assume that Assumption 7 is satisfied; then error system (9) achieves global stable situation under the designed sampled-data feedback controller (7) with the control law as follows:

$$
\begin{aligned}
K_{i}^{2} & \leq \min \left\{\Xi_{1}, \Xi_{2}\right\}, \\
K_{j}^{2} & \leq \min \left\{\Pi_{1}, \Pi_{2}\right\}, \\
\lambda_{i} & >\sum_{i=1}^{n}\left[\left|\check{d}_{i}-\widehat{d}_{i}\right| T_{i}+\sum_{j=1}^{m}\left|\check{a}_{j i}-\widehat{a}_{j i}\right| L_{y} R_{j}\right. \\
& \left.+\sum_{j=1}^{m}\left|\check{b}_{j i}-\widehat{b}_{j i}\right| \Gamma_{j}+\sum_{j=1}^{m}\left|\check{c}_{j i}-c_{j i}\right| \mu L_{y} R_{j}\right], \\
\lambda_{j} & >\sum_{i=1}^{n}\left[\left|\check{p}_{j}-\widehat{p}_{j}\right| R_{j}+\sum_{i=1}^{n}\left|\check{m}_{i j}-\widehat{m}_{i j}\right| L_{x} T_{i}\right. \\
& \left.+\sum_{i=1}^{n}\left|\check{n}_{i j}-\widehat{n}_{i j}\right| \Delta_{i}+\sum_{i=1}^{n}\left|\check{q}_{i j}-\widehat{q}_{i j}\right| \varepsilon L_{x} T_{i}\right],
\end{aligned}
$$

where

$$
\begin{aligned}
& L_{x}= \max \left\{\left|\alpha_{i}\right|,\left|\beta_{i}\right|\right\}, \\
& L_{y}= \max \left\{\left|\alpha_{j}\right|,\left|\beta_{j}\right|\right\}, \\
& \Xi_{1}= 2 \check{d}_{i}-\sum_{j=1}^{m}\left(\check{a}_{j i}^{2} L_{y}^{2}+\check{b}_{j i}^{2} L_{y}^{2}+\check{c}_{j i}^{2}\right)-\frac{1}{1-d_{1}^{\prime}}-J_{1} \\
&-m \varepsilon L_{x}^{2}-\frac{1+J_{2}}{1-\sigma_{1}}, \\
& \Xi_{2}= 2 \widehat{d}_{i}-\sum_{j=1}^{m}\left(\widehat{a}_{j i}^{2} L_{y}^{2}+\widehat{b}_{j i}^{2} L_{y}^{2}+\widehat{c}_{j i}^{2}\right)-\frac{1}{1-d_{1}^{\prime}}-J_{1} \\
&-m \varepsilon L_{x}^{2}-\frac{1+J_{2}}{1-\sigma_{1}}, \\
& \Pi_{1}= 2 \check{p}_{j}-\sum_{i=1}^{n}\left(\check{m}_{i j}^{2} L_{x}^{2}+\check{n}_{i j}^{2} L_{x}^{2}+\check{q}_{i j}^{2}\right)-\frac{1}{1-d_{2}^{\prime}}-H_{1} \\
&-n \mu L_{y}^{2}-\frac{1+H_{2}}{1-\tau_{1}}, \\
& \Pi_{2}= 2 \widehat{p}_{j}-\sum_{i=1}^{n}\left(\widehat{m}_{i j}^{2} L_{x}^{2}+\widehat{n}_{i j}^{2} L_{x}^{2}+\widehat{q}_{i j}^{2}\right)-\frac{1}{1-d_{2}^{\prime}}-H_{1} \\
& L_{y}-\frac{1+H_{2}}{1-\tau_{1}}, \\
&
\end{aligned}
$$

and $J_{1}, J_{2}, H_{1}, H_{2}$ are all positive constants. 
Proof. Consider the following Lyapunov function for synchronization error system (9):

$$
V(t)=V_{x}(t)+V_{y}(t)
$$

where

$$
\begin{aligned}
V_{x}(t)= & \frac{1}{2} \sum_{i=1}^{n} e_{i}^{T}(t) e_{i}(t) \\
& +\sum_{i=1}^{n} \frac{1}{2\left(1-d_{1}^{\prime}\right)} \int_{t-d_{1}(t)}^{t} e_{i}^{T}(s) e_{i}(s) d s \\
& +\sum_{j=1}^{m} \frac{1+H_{2}}{2\left(1-\tau_{1}\right)} \int_{t-\tau(t)}^{t} e_{j}^{T}(s) e_{j}(s) d s \\
& +\frac{1}{2} \sum_{i=1}^{n} \sum_{j=1}^{m} \int_{-\mu}^{0} \int_{t+s}^{t} e_{j}^{T}(z) L_{y}^{T} L_{y} e_{j}(z) d z d s, \\
V_{y}(t)= & \frac{1}{2} \sum_{j=1}^{m} e_{j}^{T}(t) e_{j}(t) \\
& +\sum_{j=1}^{m} \frac{1}{2\left(1-d_{2}^{\prime}\right)} \int_{t-d_{2}(t)}^{t} e_{j}^{T}(s) e_{j}(s) d s \\
& +\sum_{i=1}^{n} \frac{1+J_{2}}{2\left(1-\sigma_{1}\right)} \int_{t-\sigma(t)}^{t} e_{i}^{T}(s) e_{i}(s) d s \\
& +\frac{1}{2} \sum_{i=1}^{n} \sum_{j=1}^{m} \int_{-\varepsilon}^{0} \int_{t+s}^{t} e_{i}^{T}(z) L_{x}^{T} L_{x} e_{i}(z) d z d s .
\end{aligned}
$$

Case 1. If $\left|x_{i}(t)\right| \leq T_{i},\left|\widehat{x}_{i}(t)\right| \leq T_{i},\left|y_{j}(t)\right| \leq R_{j},\left|\widehat{y}_{j}(t)\right| \leq R_{j}$ at time $t$, according to the jumping rules, systems (3) and (5) are reduced to systems (28) and (29), respectively:

$$
\begin{aligned}
& d x_{i}(t)=\left[-\check{d}_{i} x_{i}(t)+\sum_{j=1}^{m} \check{a}_{j i} f_{j}\left(y_{j}(t)\right)\right. \\
& +\sum_{j=1}^{m} \check{b}_{j i} f_{j}\left(y_{j}(t-\tau(t))\right) \\
& \left.+\sum_{j=1}^{m} \check{c}_{j i} \int_{t-\mu(t)}^{t} f_{j}\left(y_{j}(s)\right) d s+I_{i}(t)\right] d t \\
& +\sum_{j=1}^{m} \beta_{j i}\left(t, y_{j}(t), y_{j}(t-\tau(t))\right) d \omega_{j}(t), \\
& d y_{j}(t)=\left[-\check{p}_{j} y_{j}(t)+\sum_{i=1}^{n} \check{m}_{i j} g_{i}\left(x_{i}(t)\right)\right. \\
& +\sum_{i=1}^{n} \check{n}_{i j} g_{i}\left(x_{i}(t-\sigma(t))\right)
\end{aligned}
$$

$$
\begin{aligned}
& \left.+\sum_{i=1}^{n} \check{q}_{i j} \int_{t-\varepsilon(t)}^{t} g_{i}\left(x_{i}(s)\right) d s+I_{j}(t)\right] d t \\
& +\sum_{i=1}^{n} \beta_{i j}\left(t, x_{i}(t), x_{i}(t-\sigma(t))\right) d \omega_{i}(t) .
\end{aligned}
$$

Then we define the corresponding response system

$$
\begin{aligned}
& d \widehat{x}_{i}(t)=\left[-\check{d}_{i} \widehat{x}_{i}(t)+\sum_{j=1}^{m} \check{a}_{j i} f_{j}\left(\widehat{y}_{j}(t)\right)\right. \\
& +\sum_{j=1}^{m} \check{b}_{j i} f_{j}\left(\widehat{y}_{j}(t-\tau(t))\right)+I_{i}(t)+U_{i}(t) \\
& \left.+\sum_{j=1}^{m} \check{c}_{j i} \int_{t-\mu(t)}^{t} f_{j}\left(\widehat{y}_{j}(s)\right) d s\right] d t \\
& +\sum_{j=1}^{m} \beta_{j i}\left(t, \widehat{y}_{j}(t), \widehat{y}_{j}(t-\tau(t))\right) d \omega_{j}(t), \\
& d \widehat{y}_{j}(t)=\left[-\check{p}_{j} \widehat{y}_{j}(t)+\sum_{i=1}^{n} \check{m}_{i j} g_{i}\left(\widehat{x}_{i}(t)\right)\right. \\
& +\sum_{i=1}^{n} \check{n}_{i j} g_{i}\left(\widehat{x}_{i}(t-\sigma(t))\right)+I_{j}(t)+U_{j}(t) \\
& \left.+\sum_{i=1}^{n} \check{q}_{i j} \int_{t-\varepsilon(t)}^{t} g_{i}\left(\widehat{x}_{i}(s)\right) d s\right] d t \\
& +\sum_{i=1}^{n} \beta_{i j}\left(t, \widehat{x}_{i}(t), \widehat{x}_{i}(t-\sigma(t))\right) d \omega_{i}(t) .
\end{aligned}
$$

And error system (9) can be rewritten:

$$
\begin{aligned}
& d e_{i}(t)=d \widehat{x}_{i}(t)-d x_{i}(t)=\left[-\check{d}_{i} \widehat{x}_{i}(t)\right. \\
& +\sum_{j=1}^{m} \check{a}_{j i}\left(\widehat{x}_{i}(t)\right) f_{j}\left(\widehat{y}_{j}(t)\right) \\
& +\sum_{j=1}^{m} \check{b}_{j i} f_{j}\left(\hat{y}_{j}(t-\tau(t))\right)+I_{i}(t)+U_{i}(t) \\
& +\sum_{j=1}^{m} \check{c}_{j i} \int_{t-\mu(t)}^{t} f_{j}\left(\widehat{y}_{j}(s)\right) d s+\check{d}_{i} x_{i}(t) \\
& \quad-\sum_{j=1}^{m} \check{a}_{j i} f_{j}\left(y_{j}(t)\right)-\sum_{j=1}^{m} \check{b}_{j i} f_{j}\left(y_{j}(t-\tau(t))\right)
\end{aligned}
$$


8

Mathematical Problems in Engineering

$$
\begin{aligned}
& \left.-\sum_{j=1}^{m} \check{c}_{j i} \int_{t-\mu(t)}^{t} f_{j}\left(y_{j}(s)\right) d s\right] d t \\
& +\sum_{j=1}^{m} \beta_{j i}\left(t, e_{j}(t), e_{j}(t-\tau(t))\right) d \omega_{j}(t) .
\end{aligned}
$$

Then

$$
\begin{aligned}
& d e_{i}(t)=\left[-\check{d}_{i} e_{i}(t)+\sum_{j=1}^{m} \check{a}_{j i} F_{j}\left(e_{j}(t)\right)\right. \\
& +\sum_{j=1}^{m} \check{b}_{j i} F_{j}\left(e_{j}(t-\tau(t))\right) \\
& +\sum_{j=1}^{m} \check{c}_{j i} \int_{t-\mu(t)}^{t} F_{j}\left(e_{j}(s)\right) d s+K_{i} e_{i}\left(t-d_{1}(t)\right) \\
& \left.-\lambda_{i} \operatorname{sign}\left(e_{i}(t)\right)\right] d t \\
& +\sum_{j=1}^{m} \beta_{j i}\left(t, e_{j}(t), e_{j}(t-\tau(t))\right) d \omega_{j}(t) .
\end{aligned}
$$

We conclude that

$$
\begin{aligned}
& d e_{j}(t)=d \widehat{y}_{j}(t)-d y_{j}(t)=\left[-\check{p}_{j} e_{j}(t)\right. \\
& +\sum_{i=1}^{n} \check{m}_{i j} G_{i}\left(e_{i}(t)\right)+\sum_{i=1}^{n} \check{n}_{i j} G_{i} e_{i}(t-\sigma(t)) \\
& +\sum_{i=1}^{n} \check{q}_{i j} \int_{t-\varepsilon(t)}^{t} G_{i}\left(e_{i}(s)\right) d s+K_{j} e_{j}\left(t-d_{2}(t)\right) \\
& \left.-\lambda_{j} \operatorname{sign}\left(e_{j}(t)\right)\right] d t \\
& +\sum_{i=1}^{n} \beta_{i j}\left(t, e_{i}(t), e_{i}(t-\sigma(t))\right) d \omega_{i}(t),
\end{aligned}
$$

where

$$
\begin{aligned}
F_{j}\left(e_{j}(t)\right)= & f_{j}\left(\hat{y}_{j}(t)\right)-f_{j}\left(y_{j}(t)\right), \\
F_{j}\left(e_{j}(t-\tau(t))\right)= & f_{j}\left(\widehat{y}_{j}(t-\tau(t))\right) \\
& -f_{j}\left(y_{j}(t-\tau(t))\right), \\
G_{i}\left(e_{i}(t)\right)= & g_{i}\left(\hat{x}_{i}(t)\right)-g_{i}\left(x_{i}(t)\right), \\
G_{i}\left(e_{i}(t-\sigma(t))\right)= & g_{i}\left(\hat{x}_{i}(t-\sigma(t))\right) \\
& -g_{i}\left(x_{i}(t-\sigma(t))\right) .
\end{aligned}
$$

From all the above discussion, we get

$$
\begin{gathered}
\dot{V}_{x}(t)=\sum_{i=1}^{n} e_{i}(t) \dot{e}_{i}(t)+\sum_{j=1}^{m}\left[\frac{1+H_{2}}{2\left(1-\tau_{1}\right)} e_{j}^{2}(t)-\frac{1+H_{2}}{2}\right. \\
\left.\cdot e_{j}^{2}(t-\tau(t))\right]+\sum_{i=1}^{n}\left[\frac{1}{2\left(1-d_{1}^{\prime}\right)} e_{i}^{2}(t)-\frac{1}{2} e_{i}^{2}(t\right. \\
\left.\left.-d_{1}(t)\right)\right]+\frac{1}{2} \sum_{i=1}^{n} \sum_{j=1}^{m}\left[\int_{-\mu}^{0} e_{j}^{T}(t) L_{y}^{T} L_{y} e_{j}(t) d s\right. \\
\left.-\int_{-\mu}^{0} e_{j}^{T}(t+s) L_{y}^{T} L_{y} e_{j}(t+s) d s\right]
\end{gathered}
$$

and then

$$
\begin{gathered}
\dot{V}_{x}(t) \leq \sum_{i=1}^{n} e_{i}(t) \dot{e}_{i}(t)+\sum_{j=1}^{m}\left[\frac{1+H_{2}}{2\left(1-\tau_{1}\right)} e_{j}^{2}(t)-\frac{1+H_{2}}{2}\right. \\
\left.\cdot e_{j}^{2}(t-\tau(t))\right]+\sum_{i=1}^{n}\left[\frac{1}{2\left(1-d_{1}^{\prime}\right)} e_{i}^{2}(t)-\frac{1}{2} e_{i}^{2}(t\right. \\
\left.\left.-d_{1}(t)\right)\right]+\frac{1}{2} \sum_{i=1}^{n} \sum_{j=1}^{m}\left[\mu e_{j}^{T}(t) L_{y}^{T} L_{y} e_{j}(t)\right. \\
\left.-\int_{t-\mu(t)}^{t} e_{j}^{T}(s) L_{y}^{T} L_{y} e_{j}(s) d s\right] .
\end{gathered}
$$

According to (31), one has

$$
\begin{aligned}
& \dot{V}_{x}(t)=\sum_{i=1}^{n} e_{i}(t)\left\{-\check{d}_{i} e_{i}(t)+\sum_{j=1}^{m} \check{a}_{j i} F_{j}\left(e_{j}(t)\right)\right. \\
& +\sum_{j=1}^{m} \check{b}_{j i} F_{j}\left(e_{j}(t-\tau(t))\right)-\lambda_{i} \operatorname{sign}\left(e_{i}(t)\right)+\sum_{j=1}^{m} \check{c}_{j i} \\
& \left.\cdot \int_{t-\mu(t)}^{t} F_{j}\left(e_{j}(s)\right) d s+K_{i} e_{i}\left(t-d_{1}(t)\right)\right\}+\frac{1}{2} \\
& \cdot \sum_{j=1}^{m} \operatorname{Tr}\left[\beta_{j i}^{T}\left(t, e_{j}(t), e_{j}(t-\tau(t))\right)\right. \\
& \left.\cdot \beta_{j i}\left(t, e_{j}(t), e_{j}(t-\tau(t))\right)\right]+\sum_{j=1}^{m}\left[\frac{1+H_{2}}{2\left(1-\tau_{1}\right)}\right. \\
& \left.\cdot \int_{t-\mu(t)}^{t} e_{j}^{T}(s) L_{y}^{T} L_{y} e_{j}(s) d s\right] \cdot \\
& \left.\cdot \sum_{i=1}^{n} \sum_{j=1}^{m}(t)-\frac{1+H_{2}}{2} e_{j}^{2}(t-\tau(t))\right]+\sum_{i=1}^{n}\left[\frac{1}{2\left(1-d_{1}^{\prime}\right)}\right. \\
& e_{j}^{T}(t) L_{y}^{T} L_{y} e_{j}(t) \\
& \left.\left.\quad d_{1}(t)\right)\right]+\frac{1}{2}
\end{aligned}
$$


Mathematical Problems in Engineering

9

Due to Assumptions 7 and 8, we obtain the following inequality:

$$
\begin{aligned}
\dot{V}_{x}(t) & \leq \sum_{i=1}^{n} e_{i}(t)\left\{-\check{d}_{i} e_{i}(t)+\sum_{j=1}^{m} \check{a}_{j i} F_{j}\left(e_{j}(t)\right)\right. \\
& +\sum_{j=1}^{m} \check{b}_{j i} F_{j}\left(e_{j}(t-\tau(t))\right)-\lambda_{i} \operatorname{sign}\left(e_{i}(t)\right)+\sum_{j=1}^{m} \check{c}_{j i} \\
& \left.\cdot \int_{t-\mu(t)}^{t} F_{j}\left(e_{j}(s)\right) d s+K_{i} e_{i}\left(t-d_{1}(t)\right)\right\}+\frac{1}{2} \\
& \cdot \sum_{j=1}^{m}\left[e_{j}^{T}(t) H_{1} e_{j}(t)+e_{j}^{T}(t-\tau(t)) H_{2} e_{j}(t-\tau(t))\right] \\
+ & \sum_{j=1}^{m}\left[\frac{1+H_{2}}{2\left(1-\tau_{1}\right)} e_{j}^{2}(t)-\frac{1+H_{2}}{2} e_{j}^{2}(t-\tau(t))\right] \\
+ & \sum_{i=1}^{n}\left[\frac{1}{2\left(1-d_{1}^{\prime}\right)} e_{i}^{2}(t)-\frac{1}{2} e_{i}^{2}\left(t-d_{1}(t)\right)\right]+\frac{1}{2} \\
& +\sum_{i=1}^{n} \sum_{j=1}^{m}\left[\mu e_{j}^{T}(t) L_{y}^{T} L_{y} e_{j}(t)\right. \\
& \left.-\int_{t-\mu(t)}^{t} e_{j}^{T}(s) L_{y}^{T} L_{y} e_{j}(s) d s\right],
\end{aligned}
$$

and then

$$
\begin{aligned}
& \dot{V}_{x}(t) \leq \sum_{i=1}^{n}\left\{-\check{d}_{i} e_{i}^{2}(t)+\sum_{j=1}^{m}\left|\check{a}_{j i}\right|\left|e_{i}(t)\right| L_{y}\left|e_{j}(t)\right|\right. \\
& +\sum_{j=1}^{m}\left|\check{b}_{j i}\right|\left|e_{i}(t)\right| L_{y}\left|e_{j}(t-\tau(t))\right|+\sum_{j=1}^{m}\left|\check{c}_{j i}\right|\left|e_{i}(t)\right| \\
& \cdot \int_{t-\mu(t)}^{t} L_{y}\left|e_{j}(s)\right| d s+K_{i}\left|e_{i}(t)\right|\left|e_{i}\left(t-d_{1}(t)\right)\right| \\
& \left.-\lambda_{i}\left|e_{i}(t)\right|\right\}+\frac{1}{2} \sum_{j=1}^{m}\left[e_{j}^{T}(t) H_{1} e_{j}(t)+e_{j}^{T}(t-\tau(t))\right. \\
& \left.\cdot H_{2} e_{j}(t-\tau(t))\right]+\sum_{j=1}^{m}\left[\frac{1+H_{2}}{2\left(1-\tau_{1}\right)} e_{j}^{2}(t)-\frac{1+H_{2}}{2}\right. \\
& \left.\cdot e_{j}^{2}(t-\tau(t))\right]+\sum_{i=1}^{n}\left[\frac{1}{2\left(1-d_{1}^{\prime}\right)} e_{i}^{2}(t)-\frac{1}{2} e_{i}^{2}(t\right. \\
& \left.-\int_{t-\mu(t)}^{t} e_{j}^{T}(s) L_{y}^{T} L_{y} e_{j}(s) d s\right] \cdot \\
& \left.\left.-d_{1}(t)\right)\right]+\frac{1}{2} \sum_{i=1}^{n} \sum_{j=1}^{m}\left[\mu e_{j}^{T}(t) L_{y}^{T} L_{y} e_{j}(t)\right.
\end{aligned}
$$

Based on Lemma 10, we get the inequalities as follows:

$$
\begin{aligned}
& K_{i}\left|e_{i}(t)\right|\left|e_{i}\left(t-d_{1}(t)\right) \leq\right| \frac{1}{2} e_{i}^{T}(t) K_{i}^{T} K_{i} e_{i}(t) \\
& \quad+\frac{1}{2} e_{i}^{T}\left(t-d_{1}(t)\right) e_{i}\left(t-d_{1}(t)\right), \\
& \left|\check{a}_{j i}\right|\left|e_{i}(t)\right| L_{y}\left|e_{j}(t)\right| \leq \frac{1}{2} e_{i}^{T}(t) L_{y}^{T} \check{a}_{j i}^{T} \check{a}_{j i} L_{y} e_{i}(t) \\
& \quad+\frac{1}{2} e_{j}^{T}(t) e_{j}(t), \\
& \left|\check{b}_{j i}\right|\left|e_{i}(t)\right| L_{y}\left|e_{j}(t-\tau(t))\right| \leq \frac{1}{2} e_{i}^{T}(t) L_{y}^{T} \check{b}_{j i}^{T} \check{b}_{j i} L_{y} e_{i}(t) \\
& \quad+\frac{1}{2} e_{j}^{T}(t-\tau(t)) e_{j}(t-\tau(t)), \\
& \left|\check{c}_{j i}\right|\left|e_{i}(t)\right| \int_{t-\mu(t)}^{t} L_{y}\left|e_{j}(s)\right| \leq \frac{1}{2} e_{i}^{T}(t) \check{c}_{j i}^{T} \check{c}_{j i} e_{i}(t) \\
& \quad+\frac{1}{2} \int_{t-\mu(t)}^{t} e_{j}^{T}(s) L_{y}^{T} L_{y} e_{j}(s) d s .
\end{aligned}
$$

Thus, we infer that

$$
\begin{aligned}
& \dot{V}_{x}(t) \leq \sum_{i=1}^{n}\left\{-\check{d}_{i} e_{i}^{2}(t)+\frac{1}{2} \sum_{j=1}^{m} \check{a}_{j i}^{2} L_{y}^{2} e_{i}^{2}(t)+\frac{1}{2} \sum_{j=1}^{m} e_{j}^{2}(t)\right. \\
& +\frac{1}{2} \sum_{j=1}^{m} \breve{b}_{j i}^{2} L_{y}^{2} e_{i}^{2}(t)+\frac{1}{2} \sum_{j=1}^{m} e_{j}^{2}(t-\tau(t))+\frac{1}{2} \\
& \cdot \sum_{j=1}^{m} \check{c}_{j i}^{2} e_{i}^{2}(t)+\frac{1}{2} \sum_{j=1}^{m} \int_{t-\mu(t)}^{t} e_{j}^{T}(s) L_{y}^{T} L_{y} e_{j}(s) d s+\frac{1}{2} \\
& \left.\cdot K_{i}^{2} e_{i}^{2}(t)+\frac{1}{2} e_{i}^{2}\left(t-d_{1}(t)\right)-\lambda_{i}\left|e_{i}(t)\right|\right\}+\frac{1}{2} \\
& \cdot \sum_{j=1}^{m}\left[H_{1} e_{j}^{2}(t)+H_{2} e_{j}^{2}(t-\tau(t))\right]+\sum_{j=1}^{m}\left[\frac{1+H_{2}}{2\left(1-\tau_{1}\right)}\right. \\
& \left.\cdot e_{j}^{2}(t)-\frac{1+H_{2}}{2} e_{j}^{2}(t-\tau(t))\right]+\sum_{i=1}^{n}\left[\frac{1}{2\left(1-d_{1}^{\prime}\right)}\right. \\
& \left.\cdot e_{i}^{2}(t)-\frac{1}{2} e_{i}^{2}\left(t-d_{1}(t)\right)\right]+\frac{1}{2} \\
& \cdot \sum_{i=1}^{n} \sum_{j=1}^{m}\left[\mu e_{j}^{T}(t) L_{y}^{T} L_{y} e_{j}(t)\right. \\
& \left.-\int_{t-\mu(t)}^{t} e_{j}^{T}(s) L_{y}^{T} L_{y} e_{j}(s) d s\right] .
\end{aligned}
$$


After organizing the above formulas, we deduce that

$$
\begin{aligned}
& +\frac{1+J_{2}}{2\left(1-\sigma_{1}\right)} \leq 0 \\
& -\check{p}_{j}+\frac{1}{2} \sum_{i=1}^{n} \check{m}_{i j}^{2} L_{x}^{2}+\frac{1}{2} \sum_{i=1}^{n} \check{n}_{i j}^{2} L_{x}^{2}+\frac{1}{2} \sum_{i=1}^{n} \check{q}_{i j}^{2}+\frac{1}{2} K_{j}^{2} \\
& +\frac{1}{2\left(1-d_{2}^{\prime}\right)}+\frac{1}{2}+\frac{1}{2} H_{1}+\frac{n}{2} \mu L_{y}^{2} \\
& +\frac{1+H_{2}}{2\left(1-\tau_{1}\right)} \leq 0
\end{aligned}
$$$$
\dot{V}_{x}(t) \leq \sum_{i=1}^{n}\left[-\check{d}_{i}+\frac{1}{2} \sum_{j=1}^{m} \check{a}_{j i}^{2} L_{y}^{2}+\frac{1}{2} \sum_{j=1}^{m} \check{b}_{j i}^{2} L_{y}^{2}+\frac{1}{2} \sum_{j=1}^{m} \check{c}_{j i}^{2}\right.
$$

With similar process of $\dot{V}_{x}(t)$, we obtain $\dot{V}_{y}(t)$ as follows:

$$
\begin{aligned}
\dot{V}_{y}(t) & \leq \sum_{j=1}^{m}\left[-\check{p}_{j}+\frac{1}{2} \sum_{i=1}^{n} \check{m}_{i j}^{2} L_{x}^{2}+\frac{1}{2} \sum_{i=1}^{n} \check{n}_{i j}^{2} L_{x}^{2}+\frac{1}{2} \sum_{i=1}^{n} \check{q}_{i j}^{2}\right. \\
+ & \left.\frac{1}{2} K_{j}^{2}+\frac{1}{2\left(1-d_{2}^{\prime}\right)}\right] e_{j}^{2}(t)+\sum_{i=1}^{n}\left[\frac{1}{2}+\frac{1}{2} J_{1}\right. \\
+ & \left.\frac{m}{2} \varepsilon L_{x}^{2}+\frac{1+J_{2}}{2\left(1-\sigma_{1}\right)}\right] e_{i}^{2}(t) .
\end{aligned}
$$

So according to (25) and the compilation of the above equations, we have

$$
\begin{aligned}
& \dot{V}(t)=\dot{V}_{x}(t)+\dot{V}_{y}(t) \leq \sum_{i=1}^{n}\left[-\check{d}_{i}+\frac{1}{2} \sum_{j=1}^{m} \check{a}_{j i}^{2} L_{y}^{2}\right. \\
& +\frac{1}{2} \sum_{j=1}^{m} \check{b}_{j i}^{2} L_{y}^{2}+\frac{1}{2} \sum_{j=1}^{m} \check{c}_{j i}^{2}+\frac{1}{2} K_{i}^{2}+\frac{1}{2\left(1-d_{1}^{\prime}\right)}+\frac{1}{2} \\
& \left.+\frac{1}{2} J_{1}+\frac{m}{2} \varepsilon L_{x}^{2}+\frac{1+J_{2}}{2\left(1-\sigma_{1}\right)}\right] e_{i}^{2}(t)+\sum_{j=1}^{m}\left[-\check{p}_{j}\right. \\
& +\frac{1}{2} \sum_{i=1}^{n} \check{m}_{i j}^{2} L_{x}^{2}+\frac{1}{2} \sum_{i=1}^{n} \check{n}_{i j}^{2} L_{x}^{2}+\frac{1}{2} \sum_{i=1}^{n} \check{q}_{i j}^{2}+\frac{1}{2} K_{j}^{2} \\
& \left.+\frac{1}{2\left(1-d_{2}^{\prime}\right)}+\frac{1}{2}+\frac{1}{2} H_{1}+\frac{n}{2} \mu L_{y}^{2}+\frac{1+H_{2}}{2\left(1-\tau_{1}\right)}\right] \\
& +e_{j}^{2}(t) \leq 0 .
\end{aligned}
$$

According to the definitions of $K_{i}^{2}$ and $K_{j}^{2}$, one has $\dot{V}(t) \leq$ 0 . Then

$$
\begin{gathered}
-\check{d}_{i}+\frac{1}{2} \sum_{j=1}^{m} \check{a}_{j i}^{2} L_{y}^{2}+\frac{1}{2} \sum_{j=1}^{m} \check{b}_{j i}^{2} L_{y}^{2}+\frac{1}{2} \sum_{j=1}^{m} \check{c}_{j i}^{2}+\frac{1}{2} K_{i}^{2} \\
+\frac{1}{2\left(1-d_{1}^{\prime}\right)}+\frac{1}{2}+\frac{1}{2} J_{1}+\frac{m}{2} \varepsilon L_{x}^{2}
\end{gathered}
$$

Case 2. If $\left|x_{i}(t)\right|>T_{i},\left|\widehat{x}_{i}(t)\right|>T_{i},\left|y_{j}(t)\right|>R_{j},\left|\hat{y}_{j}(t)\right|>R_{j}$ at time $t$, systems (3) and (5) can be reduced to the following systems, respectively:

$$
\begin{aligned}
& d x_{i}(t)=\left[-\widehat{d}_{i} x_{i}(t)+\sum_{j=1}^{m} \widehat{a}_{j i} f_{j}\left(y_{j}(t)\right)\right. \\
& +\sum_{j=1}^{m} \widehat{b}_{j i} f_{j}\left(y_{j}(t-\tau(t))\right) \\
& \left.+\sum_{j=1}^{m} \widehat{c}_{j i} \int_{t-\mu(t)}^{t} f_{j}\left(y_{j}(s)\right) d s+I_{i}(t)\right] d t+\sum_{j=1}^{m} \beta_{j i} \\
& +\left(t, y_{j}(t), y_{j}(t-\tau(t))\right) d \omega_{j}(t), \\
& d \widehat{x}_{i}(t)=\left[-\widehat{d}_{i} \widehat{x}_{i}(t)+\sum_{j=1}^{m} \widehat{a}_{j i} f_{j}\left(\hat{y}_{j}(t)\right)\right. \\
& +\sum_{j=1}^{m} \widehat{b}_{j i} f_{j}\left(\hat{y}_{j}(t-\tau(t))\right)+I_{i}(t) \\
& \left.+\sum_{j=1}^{m} \widehat{c}_{j i} \int_{t-\mu(t)}^{t} f_{j}\left(\widehat{y}_{j}(s)\right) d s+U_{i}(t)\right] d t \\
& +\sum_{j=1}^{m} \beta_{j i}\left(t, \widehat{y}_{j}(t), \widehat{y}_{j}(t-\tau(t))\right) d \omega_{j}(t) .
\end{aligned}
$$
follows:

Then the corresponding error system can be defined as

$$
\begin{aligned}
& d e_{i}(t)=\left[-\widehat{d}_{i} e_{i}(t)+\sum_{j=1}^{m} \check{a}_{j i} F_{j}\left(e_{j}(t)\right)\right. \\
& +\sum_{j=1}^{m} \widehat{b}_{j i} F_{j}\left(e_{j}(t-\tau(t))\right) \\
& +\sum_{j=1}^{m} \widehat{c}_{j i} \int_{t-\mu(t)}^{t} F_{j}\left(e_{j}(s)\right) d s-\lambda_{i} \operatorname{signe}_{i}(t)
\end{aligned}
$$




$$
\begin{aligned}
& \left.+K_{i} e_{i}\left(t-d_{1}(t)\right)\right] d t \\
& +\sum_{j=1}^{m} \beta_{j i}\left(t, e_{j}(t), e_{j}(t-\tau(t))\right) d \omega_{j}(t) .
\end{aligned}
$$

The following line is similar to $\dot{V}_{x}(t)$ of Case 1 , and we obtain $\dot{V}_{y}(t)$ and $\dot{V}(t)$ as follows:

$$
\begin{aligned}
& \dot{V}(t)=\dot{V}_{x}(t)+\dot{V}_{y}(t) \leq \sum_{i=1}^{n}\left[-\widehat{d}_{i}+\frac{1}{2} \sum_{j=1}^{m} \widehat{a}_{j i}^{2} L_{y}^{2}\right. \\
& +\frac{1}{2} \sum_{j=1}^{m} \widehat{b}_{j i}^{2} L_{y}^{2}+\frac{1}{2} \sum_{j=1}^{m} \widehat{c}_{j i}^{2}+\frac{1}{2} K_{i}^{2}+\frac{1}{2\left(1-d_{1}^{\prime}\right)}+\frac{1}{2} \\
& \left.+\frac{1}{2} J_{1}+\frac{m}{2} \varepsilon L_{x}^{2}+\frac{1+J_{2}}{2\left(1-\sigma_{1}\right)}\right] e_{i}^{2}(t)+\sum_{j=1}^{m}\left[-\widehat{p}_{j}\right. \\
& +\frac{1}{2} \sum_{i=1}^{n} \widehat{m}_{i j}^{2} L_{x}^{2}+\frac{1}{2} \sum_{i=1}^{n} \widehat{n}_{i j}^{2} L_{x}^{2}+\frac{1}{2} \sum_{i=1}^{n} \widehat{q}_{i j}^{2}+\frac{1}{2} K_{j}(t) \\
& \left.+\frac{1}{2\left(1-d_{2}^{\prime}\right)}+\frac{1}{2}+\frac{1}{2} H_{1}+\frac{n}{2} \mu L_{y}^{2}+\frac{1+H_{2}}{2\left(1-\tau_{1}\right)}\right] \\
& +e_{j}^{2}(t) \leq 0 .
\end{aligned}
$$

According to the definitions of $K_{i}^{2}$ and $K_{j}^{2}$, one has $\dot{V}(t) \leq$ 0 . Thus

$$
\begin{aligned}
& -\widehat{d}_{i}+\frac{1}{2} \sum_{j=1}^{m} \widehat{a}_{j i}^{2} L_{y}^{2}+\frac{1}{2} \sum_{j=1}^{m} \widehat{b}_{j i}^{2} L_{y}^{2}+\frac{1}{2} \sum_{j=1}^{m} \widehat{c}_{j i}^{2}+\frac{1}{2} K_{i}^{2} \\
& +\frac{1}{2\left(1-d_{1}^{\prime}\right)}+\frac{1}{2}+\frac{1}{2} J_{1}+\frac{m}{2} \varepsilon L_{x}^{2} \\
& +\frac{1+J_{2}}{2\left(1-\sigma_{1}\right)} \leq 0, \\
& -\widehat{p}_{j}+\frac{1}{2} \sum_{i=1}^{n} \widehat{m}_{i j}^{2} L_{x}^{2}+\frac{1}{2} \sum_{i=1}^{n} \widehat{n}_{i j}^{2} L_{x}^{2}+\frac{1}{2} \sum_{i=1}^{n} \widehat{q}_{i j}^{2}+\frac{1}{2} K_{j}^{2} \\
& +\frac{1}{2\left(1-d_{2}^{\prime}\right)}+\frac{1}{2}+\frac{1}{2} H_{1}+\frac{n}{2} \mu L_{y}^{2} \\
& +\frac{1+H_{2}}{2\left(1-\tau_{1}\right)} \leq 0 .
\end{aligned}
$$

Case 3. If $\left|x_{i}(t)\right| \leq T_{i},\left|\widehat{x}_{i}(t)\right|>T_{i},\left|y_{j}(t)\right| \leq R_{j},\left|\hat{y}_{j}(t)\right|>$ $R_{j}$ at time $t$, on the basis of memristive connection weights, systems (3) and (5) can be rewritten as the following systems, respectively:

$$
\begin{aligned}
& d x_{i}(t)=\left[-\widehat{d}_{i} x_{i}(t)+\sum_{j=1}^{m} \widehat{a}_{j i} f_{j}\left(y_{j}(t)\right)\right. \\
& +\sum_{j=1}^{m} \widehat{b}_{j i} f_{j}\left(y_{j}(t-\tau(t))\right) \\
& \left.+\sum_{j=1}^{m} \widehat{c}_{j i} \int_{t-\mu(t)}^{t} f_{j}\left(y_{j}(s)\right) d s+I_{i}(t)\right] d t+\sum_{j=1}^{m} \beta_{j i} \\
& \quad \cdot\left(t, y_{j}(t), y_{j}(t-\tau(t))\right) d \omega_{j}(t) .
\end{aligned}
$$

Therefore the corresponding response system can be described as

$$
\begin{aligned}
& d \widehat{x}_{i}(t)=\left[-\check{d}_{i} \widehat{x}_{i}(t)+\sum_{j=1}^{m} \check{a}_{j i} f_{j}\left(\hat{y}_{j}(t)\right)\right. \\
& +\sum_{j=1}^{m} \check{b}_{j i} f_{j}\left(\hat{y}_{j}(t-\tau(t))\right)+I_{i}(t) \\
& \left.+\sum_{j=1}^{m} \check{c}_{j i} \int_{t-\mu(t)}^{t} f_{j}\left(\hat{y}_{j}(s)\right) d s+U_{i}(t)\right] d t \\
& +\sum_{j=1}^{m} \beta_{j i}\left(t, \hat{y}_{j}(t), \hat{y}_{j}(t-\tau(t))\right) d \omega_{j}(t) .
\end{aligned}
$$

We define the error system

$$
\begin{aligned}
& d e_{i}(t)=\left[-\widehat{d}_{i} e_{i}(t)+\sum_{j=1}^{m} \widehat{a}_{j i} F_{j}\left(e_{j}(t)\right)\right. \\
& +\sum_{j=1}^{m} \widehat{b}_{j i} F_{j}\left(e_{j}(t-\tau(t))\right)+\left(\widehat{d}_{i}-\check{d}_{i}\right) \widehat{x}_{i}(t) \\
& +\sum_{j=1}^{m} \widehat{c}_{j i} \int_{t-\mu(t)}^{t} F_{j}\left(e_{j}(s)\right) d s \\
& +\sum_{j=1}^{m}\left(\check{a}_{j i}-\widehat{a}_{j i}\right) f_{j}\left(\widehat{y}_{j}(t)\right) \\
& +\sum_{j=1}^{m}\left(\check{b}_{j i}-\widehat{b}_{j i}\right) f_{j}\left(\widehat{y}_{j}(t-\tau(t))\right)
\end{aligned}
$$


12

Mathematical Problems in Engineering

$$
\begin{aligned}
& +\sum_{j=1}^{m}\left(\check{c}_{j i}-\widehat{c}_{j i}\right) \int_{t-\mu(t)}^{t} f_{j}\left(\widehat{y}_{j}(s)\right) d s \\
& \left.+K_{i} e_{i}\left(t-d_{1}(t)\right)-\lambda_{i} \operatorname{sign}\left(e_{i}(t)\right)\right] d t \\
& +\sum_{j=1}^{m} \beta_{j i}\left(t, e_{j}(t), e_{j}(t-\tau(t))\right) d \omega_{j}(t) .
\end{aligned}
$$

We infer the following inequality through (26):

$$
\begin{aligned}
& \dot{V}_{x}(t) \leq \sum_{i=1}^{n} e_{i}(t) \dot{e}_{i}(t)+\sum_{j=1}^{m}\left[\frac{1+H_{2}}{2\left(1-\tau_{1}\right)} e_{j}^{2}(t)-\frac{1+H_{2}}{2}\right. \\
& \left.\cdot e_{j}^{2}(t-\tau(t))\right]+\sum_{i=1}^{n}\left[\frac{1}{2\left(1-d_{1}^{\prime}\right)} e_{i}^{2}(t)-\frac{1}{2} e_{i}^{2}(t\right. \\
& \left.\left.-d_{1}(t)\right)\right]+\frac{1}{2} \sum_{i=1}^{n} \sum_{j=1}^{m}\left[\mu e_{j}^{T}(t) L_{\mathrm{y}}^{T} L_{y} e_{j}(t)\right. \\
& \left.-\int_{t-\mu(t)}^{t} e_{j}^{T}(s) L_{y}^{T} L_{y} e_{j}(s) d s\right] .
\end{aligned}
$$

According to (52), we have

$$
\begin{aligned}
& \dot{V}_{x}(t)=\sum_{i=1}^{n} e_{i}(t)\left\{-\widehat{d}_{i} e_{i}(t)+\sum_{j=1}^{m} \widehat{a}_{j i} F_{j}\left(e_{j}(t)\right)\right. \\
& +\sum_{j=1}^{m} \widehat{b}_{j i} F_{j}\left(e_{j}(t-\tau(t))\right)+\sum_{j=1}^{m} \widehat{c}_{j i} \\
& \cdot \int_{t-\mu(t)}^{t} F_{j}\left(e_{j}(s)\right) d s+\left(\widehat{d}_{i}-\check{d}_{i}\right) \widehat{x}_{i}(t)+\sum_{j=1}^{m}\left(\check{a}_{j i}\right. \\
& \left.-\widehat{a}_{j i}\right) f_{j}\left(\widehat{y}_{j}(t)\right)+\sum_{j=1}^{m}\left(\check{b}_{j i}-\widehat{b}_{j i}\right) f_{j}\left(\widehat{y}_{j}(t-\tau(t))\right) \\
& +\sum_{j=1}^{m}\left(\check{c}_{j i}-\widehat{c}_{j i}\right) \int_{t-\mu(t)}^{t} f_{j}\left(\widehat{y}_{j}(s)\right) d s+K_{i} e_{i}(t \\
& \left.\left.-d_{1}(t)\right)-\lambda_{i} \operatorname{sign}\left(e_{i}(t)\right)\right\}+\frac{1}{2} \\
& \cdot \sum_{j=1}^{m} \operatorname{Tr}\left[\beta_{j i}^{T}\left(t, e_{j}(t), e_{j}(t-\tau(t))\right)\right. \\
& \left.\times \beta_{j i}\left(t, e_{j}(t), e_{j}(t-\tau(t))\right)\right]+\sum_{j=1}^{m}\left[\frac{1+H_{2}}{2\left(1-\tau_{1}\right)}\right. \\
& \left.\cdot e_{j}^{2}(t)-\frac{1+H_{2}}{2} e_{j}^{2}(t-\tau(t))\right]+\sum_{i=1}^{n}\left[\frac{1}{2\left(1-d_{1}^{\prime}\right)}\right.
\end{aligned}
$$

$$
\begin{aligned}
& \left.\cdot e_{i}^{2}(t)-\frac{1}{2} e_{i}^{2}\left(t-d_{1}(t)\right)\right]+\frac{1}{2} \\
& \cdot \sum_{i=1}^{n} \sum_{j=1}^{m}\left[\mu e_{j}^{T}(t) L_{y}^{T} L_{y} e_{j}(t)\right. \\
& \left.-\int_{t-\mu(t)}^{t} e_{j}^{T}(s) L_{y}^{T} L_{y} e_{j}(s) d s\right] .
\end{aligned}
$$

Through Assumptions 7 and 8 and Lemma 10, we get

$$
\begin{aligned}
& \dot{V}_{x}(t) \leq \sum_{i=1}^{n}\left[-\widehat{d}_{i}+\frac{1}{2} \sum_{j=1}^{m} \widehat{a}_{j i}^{2} L_{y}^{2}+\frac{1}{2} \sum_{j=1}^{m} \widehat{b}_{j i}^{2} L_{y}^{2}+\frac{1}{2} \sum_{j=1}^{m} \widehat{c}_{j i}^{2}\right. \\
& \left.+\frac{1}{2} K_{i}^{2}+\frac{1}{2\left(1-d_{1}^{\prime}\right)}\right] e_{i}^{2}(t)+\sum_{j=1}^{m}\left[\frac{1}{2} H_{1}+\frac{n}{2} \mu L_{y}^{2}\right. \\
& \left.+\frac{1+H_{2}}{2\left(1-\tau_{1}\right)}\right] e_{j}^{2}(t)+\sum_{i=1}^{n}\left|e_{i}(t)\right|\left\{\left|\widehat{d}_{i}-\check{d}_{i}\right|\left|\widehat{x}_{i}(t)\right|\right. \\
& +\sum_{j=1}^{m}\left|\check{a}_{j i}-\widehat{a}_{j i}\right|\left|f_{j}\left(\widehat{y}_{j}(t)\right)\right| \\
& +\sum_{j=1}^{m}\left|\check{b}_{j i}-\widehat{b}_{j i}\right|\left|f_{j}\left(\widehat{y}_{j}(t-\tau(t))\right)\right| \\
& \left.+\sum_{j=1}^{m}\left|\check{c}_{j i}-\widehat{c}_{j i}\right|\left|\int_{t-\mu(t)}^{t} f_{j}\left(\widehat{y}_{j}(s)\right) d s\right|-\lambda_{i}\right\} .
\end{aligned}
$$

According to Assumption 7 and switching jumps, we obtain

$$
\begin{aligned}
\left|f_{j}\left(\hat{y}_{j}(t)\right)\right| & \leq L_{y} R_{j}, \\
\left|f_{j}\left(\hat{y}_{j}(t-\tau(t))\right)\right| & \leq \Gamma_{j}, \\
\int_{t-\mu(t)}^{t}\left|f_{j}\left(\hat{y}_{j}(s)\right) d s\right| & \leq \int_{t-\mu(t)}^{t} L_{y} R_{j} d s=L_{y} R_{j} \mu(t) \\
& \leq L_{y} R_{j} \mu .
\end{aligned}
$$

Then, (55) can be written as

$$
\begin{gathered}
\dot{V}_{x}(t) \leq \sum_{i=1}^{n}\left[-\widehat{d}_{i}+\frac{1}{2} \sum_{j=1}^{m} \widehat{a}_{j i}^{2} L_{y}^{2}+\frac{1}{2} \sum_{j=1}^{m} \widehat{b}_{j i}^{2} L_{y}^{2}+\frac{1}{2} \sum_{j=1}^{m} \widehat{c}_{j i}^{2}\right. \\
\left.+\frac{1}{2} K_{i}^{2}+\frac{1}{2\left(1-d_{1}^{\prime}\right)}\right] e_{i}^{2}(t)+\sum_{j=1}^{m}\left[\frac{1}{2} H_{1}+\frac{n}{2} \mu L_{y}^{2}\right.
\end{gathered}
$$


Mathematical Problems in Engineering

13

$$
\begin{aligned}
& \left.+\frac{1+H_{2}}{2\left(1-\tau_{1}\right)}\right] e_{j}^{2}(t)+\sum_{i=1}^{n}\left|e_{i}(t)\right|\left\{\left|\widehat{d}_{i}-\check{d}_{i}\right| T_{i}\right. \\
& +\sum_{j=1}^{m}\left|\check{a}_{j i}-\widehat{a}_{j i}\right| L_{y} R_{j}+\sum_{j=1}^{m}\left|\check{c}_{j i}-\widehat{c}_{j i}\right| \mu L_{y} R_{j} \\
& \left.+\sum_{j=1}^{m}\left|\check{b}_{j i}-\widehat{b}_{j i}\right| \Gamma_{j}-\lambda_{i}\right\} .
\end{aligned}
$$

According to a similar process of $\dot{V}_{x}(t)$, we have $\dot{V}(t)$ be extended as follows:

$$
\begin{aligned}
\dot{V}( & t)=\dot{V}_{x}(t)+\dot{V}_{y}(t) \leq \sum_{i=1}^{n} e_{i}^{2}(t)\left[-\widehat{d}_{i}+\frac{1}{2} \sum_{j=1}^{m} \widehat{a}_{j i}^{2} L_{y}^{2}\right. \\
& +\frac{1}{2} \sum_{j=1}^{m} \widehat{b}_{j i}^{2} L_{y}^{2}+\frac{1}{2} \sum_{j=1}^{m} \widehat{c}_{j i}^{2}+\frac{1}{2} K_{i}^{2}+\frac{1}{2\left(1-d_{1}^{\prime}\right)}+\frac{1}{2} J_{1} \\
& \left.+\frac{m}{2} \varepsilon L_{x}^{2}+\frac{1+J_{2}}{2\left(1-\sigma_{1}\right)}\right]+\sum_{j=1}^{m} e_{j}^{2}(t)\left[-\check{p}_{j}+\frac{1}{2}\right. \\
& +\sum_{i=1}^{n} \widehat{m}_{i j}^{2} L_{x}^{2}+\frac{1}{2} \sum_{i=1}^{n} \widehat{n}_{i j}^{2} L_{x}^{2}+\frac{1}{2} \sum_{i=1}^{n} \check{q}_{i j}^{2}+\frac{1}{2} K_{j}^{2} \\
& \left.+\frac{1}{2\left(1-d_{2}^{\prime}\right)}+\frac{1}{2} H_{1}+\frac{n}{2} \mu L_{y}^{2}+\frac{1+H_{2}}{2\left(1-\tau_{1}\right)}\right] \\
& +\sum_{i=1}^{n}\left|e_{i}(t)\right||| \widehat{d}_{i}-\check{d}_{i}\left|T_{i}+\sum_{j=1}^{m}\right| \check{a}_{j i}-\widehat{a}_{j i} \mid L_{y} R_{j} \\
& +\sum_{j=1}^{m}\left|e_{j}(t)\right|\left[\left|\widehat{p}_{j}-\check{p}_{j}\right| R_{j}+\sum_{i=1}^{n}\left|\check{m}_{i j}-\widehat{m}_{i j}\right| L_{x} T_{i}\right. \\
& \left.+\sum_{j=1}^{m}\left|\check{c}_{j i}-\widehat{c}_{j i}\right| \mu L_{y} R_{j}+\sum_{j=1}^{m}\left|\check{b}_{j i}-\widehat{b}_{j i}\right| \Gamma_{j}-\lambda_{i}\right]
\end{aligned}
$$

According to the definitions of $K_{i}^{2}, K_{j}^{2}, \lambda_{i}$, and $\lambda_{j}$, one has $\dot{V}(t) \leq 0$. Then we get

$$
\begin{aligned}
& -\widehat{d}_{i}+\frac{1}{2} \sum_{j=1}^{m} \widehat{a}_{j i}^{2} L_{y}^{2}+\frac{1}{2} \sum_{j=1}^{m} \widehat{b}_{j i}^{2} L_{y}^{2}+\frac{1}{2} \sum_{j=1}^{m} \widehat{c}_{j i}^{2}+\frac{1}{2} K_{i}^{2} \\
& +\frac{1}{2\left(1-d_{1}^{\prime}\right)}+\frac{1}{2} J_{1}+\frac{m}{2} \varepsilon L_{x}^{2}+\frac{1+J_{2}}{2\left(1-\sigma_{1}\right)} \leq 0, \\
& -\check{p}_{j}+\frac{1}{2} \sum_{i=1}^{n} \widehat{m}_{i j}^{2} L_{x}^{2}+\frac{1}{2} \sum_{i=1}^{n} \widehat{n}_{i j}^{2} L_{x}^{2}+\frac{1}{2} \sum_{i=1}^{n} \check{q}_{i j}^{2}+\frac{1}{2} K_{j}^{2} \\
& +\frac{1}{2\left(1-d_{2}^{\prime}\right)}+\frac{1}{2} H_{1}+\frac{n}{2} \mu L_{y}^{2}+\frac{1+H_{2}}{2\left(1-\tau_{1}\right)} \leq 0,
\end{aligned}
$$

$$
\begin{aligned}
& \left|\widehat{d}_{i}-\check{d}_{i}\right| T_{i}+\sum_{j=1}^{m}\left|\check{a}_{j i}-\widehat{a}_{j i}\right| L_{y} R_{j}+\sum_{j=1}^{m}\left|\check{c}_{j i}-\widehat{c}_{j i}\right| \mu L_{y} R_{j} \\
& \quad+\sum_{j=1}^{m}\left|\check{b}_{j i}-\widehat{b}_{j i}\right| \Gamma_{j}-\lambda_{i} \leq 0, \\
& \left|\widehat{p}_{j}-\check{p}_{j}\right| R_{j}+\sum_{i=1}^{n}\left|\check{m}_{i j}-\widehat{m}_{i j}\right| L_{x} T_{i}+\sum_{i=1}^{n}\left|\check{n}_{i j}-\widehat{n}_{i j}\right| \Delta_{i} \\
& \quad+\sum_{i=1}^{n}\left|\check{q}_{i j}-\widehat{q}_{i j}\right| L_{x} T_{i} \varepsilon-\lambda_{j} \leq 0 .
\end{aligned}
$$

Case 4. If $\left|x_{i}(t)\right|>T_{i},\left|\widehat{x}_{i}(t)\right| \leq T_{i},\left|y_{j}(t)\right|>R_{j},\left|\widehat{y}_{j}(t)\right| \leq R_{j}$ at time $t$, in accordance with switching jumps, the error systems can be redefined as follows:

$$
\begin{aligned}
d e_{i}( & t)=\left[-\widehat{d}_{i} e_{i}(t)+\sum_{j=1}^{m} \widehat{a}_{j i} F_{j}\left(e_{j}(t)\right)\right. \\
& +\sum_{j=1}^{m} \widehat{b}_{j i} F_{j}\left(e_{j}(t-\tau(t))\right) \\
& +\sum_{j=1}^{m} \widehat{c}_{j i} \int_{t-\mu(t)}^{t} F_{j}\left(e_{j}(s)\right) d s+\left(\check{d}_{i}-\widehat{d}_{i}\right) \widehat{x}_{i}(t) \\
& +\sum_{j=1}^{m}\left(\widehat{a}_{j i}-\check{a}_{j i}\right) f_{j}\left(\widehat{y}_{j}(t)\right) \\
& +\sum_{j=1}^{m}\left(\widehat{b}_{j i}-\check{b}_{j i}\right) f_{j}\left(\widehat{y}_{j}(t-\tau(t))\right) \\
& +\sum_{j=1}^{m}\left(\widehat{c}_{j i}-\check{c}_{j i}\right) \int_{t-\mu(t)}^{t} f_{j}\left(\widehat{y}_{j}(s)\right) d s \\
& \left.+K_{i} e_{i}\left(t-d_{1}(t)\right)-\lambda_{i} \operatorname{sign}\left(e_{i}(t)\right)\right] d t \\
+ & \sum_{j=1}^{m} \beta_{j i}\left(t, e_{j}(t), e_{j}(t-\tau(t))\right) d \omega_{j}(t) .
\end{aligned}
$$

Similarly, evaluating $\dot{V}_{x}(t)$ along the trajectories of (60), we get $\dot{V}_{y}(t)$ and $\dot{V}(t)$.

$$
\begin{gathered}
\dot{V}(t)=\dot{V}_{x}(t)+\dot{V}_{y}(t) \leq \sum_{i=1}^{n} e_{i}^{2}(t)\left[-\widehat{d}_{i}+\frac{1}{2} \sum_{j=1}^{m} \widehat{a}_{j i}^{2} L_{y}^{2}\right. \\
+\frac{1}{2} \sum_{j=1}^{m} \widehat{b}_{j i}^{2} L_{y}^{2}+\frac{1}{2} \sum_{j=1}^{m} \widehat{c}_{j i}^{2}+\frac{1}{2} K_{i}^{2}+\frac{1}{2\left(1-d_{1}^{\prime}\right)}+\frac{1}{2} J_{1}
\end{gathered}
$$




$$
\begin{aligned}
& \left.+\frac{m}{2} \varepsilon L_{x}^{2}+\frac{1+J_{2}}{2\left(1-\sigma_{1}\right)}\right]+\sum_{j=1}^{m} e_{j}^{2}(t)\left[-\check{p}_{j}+\frac{1}{2}\right. \\
& \cdot \sum_{i=1}^{n} \widehat{m}_{i j}^{2} L_{x}^{2}+\frac{1}{2} \sum_{i=1}^{n} \widehat{n}_{i j}^{2} L_{x}^{2}+\frac{1}{2} \sum_{i=1}^{n} \check{q}_{i j}^{2}+\frac{1}{2} K_{j}^{2} \\
& \left.+\frac{1}{2\left(1-d_{2}^{\prime}\right)}+\frac{1}{2} H_{1}+\frac{n}{2} \mu L_{y}^{2}+\frac{1+H_{2}}{2\left(1-\tau_{1}\right)}\right] \\
& +\sum_{i=1}^{n}\left|e_{i}(t)\right|\left[\left|\check{d}_{i}-\widehat{d}_{i}\right| T_{i}+\sum_{j=1}^{m}\left|\widehat{a}_{j i}-\check{a}_{j i}\right| L_{y} R_{j}\right. \\
& \left.+\sum_{j=1}^{m}\left|\widehat{c}_{j i}-\check{c}_{j i}\right| \mu L_{y} R_{j}+\sum_{j=1}^{m}\left|\widehat{b}_{j i}-\check{b}_{j i}\right| \Gamma_{j}-\lambda_{i}\right] \\
& +\sum_{j=1}^{m}\left|e_{j}(t)\right|\left[\left|\check{p}_{j}-\widehat{p}_{j}\right| R_{j}+\sum_{i=1}^{n}\left|\widehat{m}_{i j}-\check{m}_{i j}\right| L_{x} T_{i}\right. \\
& \left.+\sum_{i=1}^{n}\left|\widehat{n}_{i j}-\check{n}_{i j}\right| \Delta_{i}+\sum_{i=1}^{n}\left|\widehat{q}_{i j}-\check{q}_{i j}\right| L_{x} T_{i} \varepsilon-\lambda_{j}\right] \leq 0 .
\end{aligned}
$$

According to the definitions of $K_{i}^{2}, K_{j}^{2}, \lambda_{i}$, and $\lambda_{j}$, we obtain the following estimation $\dot{V}(t) \leq 0$. So we get

$$
\begin{aligned}
& -\widehat{d}_{i}+\frac{1}{2} \sum_{j=1}^{m} \widehat{a}_{j i}^{2} L_{y}^{2}+\frac{1}{2} \sum_{j=1}^{m} \widehat{b}_{j i}^{2} L_{y}^{2}+\frac{1}{2} \sum_{j=1}^{m} \widehat{c}_{j i}^{2}+\frac{1}{2} K_{i}^{2} \\
& +\frac{1}{2\left(1-d_{1}^{\prime}\right)}+\frac{1}{2} J_{1}+\frac{m}{2} \varepsilon L_{x}^{2}+\frac{1+J_{2}}{2\left(1-\sigma_{1}\right)} \leq 0, \\
& -\check{p}_{j}+\frac{1}{2} \sum_{i=1}^{n} \widehat{m}_{i j}^{2} L_{x}^{2}+\frac{1}{2} \sum_{i=1}^{n} \widehat{n}_{i j}^{2} L_{x}^{2}+\frac{1}{2} \sum_{i=1}^{n} \check{q}_{i j}^{2}+\frac{1}{2} K_{j}^{2} \\
& +\frac{1}{2\left(1-d_{2}^{\prime}\right)}+\frac{1}{2} H_{1}+\frac{n}{2} \mu L_{y}^{2}+\frac{1+H_{2}}{2\left(1-\tau_{1}\right)} \leq 0, \\
& \left|\check{d}_{i}-\widehat{d}_{i}\right| T_{i}+\sum_{j=1}^{m}\left|\widehat{a}_{j i}-\check{a}_{j i}\right| L_{y} R_{j}-\lambda_{i} \\
& \quad+\sum_{j=1}^{m}\left|\widehat{c}_{j i}-\check{c}_{j i}\right| \mu L_{y} R_{j}+\sum_{j=1}^{m}\left|\widehat{b}_{j i}-\check{b}_{j i}\right| \Gamma_{j} \leq 0, \\
& \quad+\sum_{i=1}^{n}\left|\widehat{q}_{i j}-\check{q}_{i j}\right| L_{x} T_{i} \varepsilon+\sum_{i=1}^{n}\left|\widehat{n}_{i j}-\check{n}_{i j}\right| \Delta_{i} \leq 0 . \\
& -\widehat{p}_{j}\left|R_{j}+\sum_{i=1}^{n}\right| \widehat{m}_{i j}-\check{m}_{i j} \mid L_{x} T_{i}-\lambda{ }_{j}
\end{aligned}
$$

The proof of Theorem 11 is completed.

Remark 12. In some previous studies [23-27, 44] the authors treated the self-inhibition $d_{i}\left(x_{i}(t)\right)=1$ or $d_{i}\left(x_{i}(t)\right)=d_{i}>$ 0 . It should be mentioned that the above researches cannot refer to the case that the self-inhibition switches at two states. Because the discontinuous self-inhibition can make the system illustrate the complicated nonlinear dynamic behaviors, thus we considered the self-inhibitions $d_{i}\left(x_{i}(t)\right)$ and $p_{j}\left(y_{j}(t)\right)$ as the state switching parameters.

Corollary 13. If Assumption 7 holds, systems (31) and (32) without perturbations are asymptotically stable when the following conditions are satisfied:

$$
\begin{aligned}
K_{i}^{2} & \leq \min \left\{\Xi_{1}, \Xi_{2}\right\}, \\
K_{j}^{2} & \leq \min \left\{\Pi_{1}, \Pi_{2}\right\}, \\
\lambda_{i} & >\sum_{i=1}^{n}\left[\left|\check{d}_{i}-\widehat{d}_{i}\right| T_{i}+\sum_{j=1}^{m}\left|\check{a}_{j i}-\widehat{a}_{j i}\right| L_{y} R_{j}\right. \\
& \left.+\sum_{j=1}^{m}\left|\check{b}_{j i}-\widehat{b}_{j i}\right| \Gamma_{j}+\sum_{j=1}^{m}\left|\check{c}_{j i}-c_{j i}\right| \mu L_{y} R_{j}\right], \\
\lambda_{j} & >\sum_{i=1}^{n}\left[\left|\check{p}_{j}-\widehat{p}_{j}\right| R_{j}+\sum_{i=1}^{n}\left|\check{m}_{i j}-\widehat{m}_{i j}\right| L_{x} T_{i}\right. \\
& \left.+\sum_{i=1}^{n}\left|\check{n}_{i j}-\widehat{n}_{i j}\right| \Delta_{i}+\sum_{i=1}^{n}\left|\check{q}_{i j}-\widehat{q}_{i j}\right| \varepsilon L_{x} L_{i}\right],
\end{aligned}
$$

where

$$
\begin{aligned}
L_{x}= & \max \left\{\left|\alpha_{i}\right|,\left|\beta_{i}\right|\right\}, \\
L_{y}= & \max \left\{\left|\alpha_{j}\right|,\left|\beta_{j}\right|\right\}, \\
\Xi_{1}= & 2 \check{d}_{i}-\sum_{j=1}^{m}\left[\check{a}_{j i}^{2} L_{y}^{2}+\check{b}_{j i}^{2} L_{y}^{2}+\check{c}_{j i}^{2}\right]-\frac{1}{1-d_{1}^{\prime}}-m \varepsilon L_{x}^{2} \\
& -\frac{1}{1-\sigma_{1}}, \\
\Xi_{2}= & 2 \widehat{d}_{i}-\sum_{j=1}^{m}\left[\widehat{a}_{j i}^{2} L_{y}^{2}+\widehat{b}_{j i}^{2} L_{y}^{2}+\widehat{c}_{j i}^{2}\right]-\frac{1}{1-d_{1}^{\prime}}-m \varepsilon L_{x}^{2} \\
& -\frac{1}{1-\sigma_{1}}, \\
\Pi_{1}= & 2 \check{p}_{j}-\sum_{i=1}^{n}\left[\check{m}_{i j}^{2} L_{x}^{2}+\check{n}_{i j}^{2} L_{x}^{2}+\check{q}_{i j}^{2}\right]-\frac{1}{1-d_{2}^{\prime}} \\
& -n \mu L_{y}^{2}-\frac{1}{1-\tau_{1}}, \\
\Pi_{2}= & 2 \widehat{p}_{j}-\sum_{i=1}^{n}\left[\widehat{m}_{i j}^{2} L_{x}^{2}+\widehat{n}_{i j}^{2} L_{x}^{2}+\widehat{q}_{i j}^{2}\right]-\frac{1}{1-d_{2}^{\prime}} \\
& n L_{y}^{2}-\frac{1}{1-\tau_{1}} .
\end{aligned}
$$

Proof. The proof process is similar to Theorem 11. So the proof is omitted. 
Remark 14. Usually, we observe that the actual communication between subsystems of MBAMNNs is inevitably disturbed by the time-varying leakage delays from various uncertainties. Thus, we considered the time-varying delays in the leakage term, and then we get the asymptotic synchronization criterion for the drive and response systems.

Under Assumption 9, the drive system of MBAMNNs with mixed time-varying delays can be described by

$$
\begin{aligned}
& d x_{i}(t)=\left[-d_{i}\left(x_{i}(t)\right) x_{i}(t-\delta(t))\right. \\
& +\sum_{j=1}^{m} a_{j i}\left(x_{i}(t)\right) f_{j}\left(y_{j}(t)\right)+I_{i}(t) \\
& +\sum_{j=1}^{m} b_{j i}\left(x_{i}(t-\tau(t))\right) f_{j}\left(y_{j}(t-\tau(t))\right) \\
& \left.+\sum_{j=1}^{m} c_{j i}\left(x_{i}(t)\right) \int_{t-\mu(t)}^{t} f_{j}\left(y_{j}(s)\right) d s\right] d t \\
& +\sum_{j=1}^{m} \beta_{j i}\left(t, y_{j}(t), y_{j}(t-\tau(t))\right) d \omega_{j}(t), \\
& \left.+\sum_{i=1}^{n} q_{i j}\left(y_{j}(t)\right) \int_{t-\varepsilon(t)}^{t} g_{i}\left(x_{i}(s)\right) d s\right] d t \\
& +(t)=\left[-p_{j}\left(y_{j}(t)\right) y_{j}(t-\zeta(t))\right. \\
& +\sum_{i=1}^{n} n_{i j}\left(y_{j}(t-\sigma(t))\right) g_{i}\left(x_{i}(t-\sigma(t))\right) \\
& +m_{i j}\left(y_{j}(t)\right) g_{i}\left(x_{i}(t)\right)+I_{j}(t) \\
& \left.+x_{i}(t-\sigma(t))\right) d \omega_{i}(t) .
\end{aligned}
$$

And the corresponding response system can be defined as

$$
\begin{aligned}
& d \widehat{x}_{i}(t)=\left[-d_{i}\left(\widehat{x}_{i}(t)\right) \widehat{x}_{i}(t-\delta(t))\right. \\
& +\sum_{j=1}^{m} a_{j i}\left(\widehat{x}_{i}(t)\right) f_{j}\left(\widehat{y}_{j}(t)\right)+I_{i}(t)+U_{i}(t) \\
& +\sum_{j=1}^{m} b_{j i}\left(\widehat{x}_{i}(t-\tau(t))\right) f_{j}\left(\widehat{y}_{j}(t-\tau(t))\right) \\
& \left.+\sum_{j=1}^{m} c_{j i}\left(\widehat{x}_{i}(t)\right) \int_{t-\mu(t)}^{t} f_{j}\left(\widehat{y}_{j}(s)\right) d s\right] d t
\end{aligned}
$$

$$
\begin{aligned}
& +\sum_{j=1}^{m} \beta_{j i}\left(t, \widehat{y}_{j}(t), \widehat{y}_{j}(t-\tau(t))\right) d \omega_{j}(t), \\
& d \widehat{y}_{j}(t)=\left[-p_{j}\left(\widehat{y}_{j}(t)\right) \widehat{y}_{j}(t-\zeta(t))\right. \\
& +\sum_{i=1}^{n} m_{i j}\left(\widehat{y}_{j}(t)\right) g_{i}\left(\widehat{x}_{i}(t)\right)+I_{j}(t)+U_{j}(t) \\
& +\sum_{i=1}^{n} n_{i j}\left(\hat{y}_{j}(t-\sigma(t))\right) g_{i}\left(\widehat{x}_{i}(t-\sigma(t))\right) \\
& \left.+\sum_{i=1}^{n} q_{i j}\left(\widehat{y}_{j}(t)\right) \int_{t-\varepsilon(t)}^{t} g_{i}\left(\widehat{x}_{i}(s)\right) d s\right] d t \\
& +\sum_{i=1}^{n} \beta_{i j}\left(t, \widehat{x}_{i}(t), \widehat{x}_{i}(t-\sigma(t))\right) d \omega_{i}(t) .
\end{aligned}
$$

Corollary 15. Suppose Assumption 7 is satisfied, then systems (65) and (66) globally achieve synchronization under designed sampled-data feedback controller (7) with the control law as follows:

$$
\begin{aligned}
K_{i}^{2} & \leq \min \left\{\Xi_{1}, \Xi_{2}\right\}, \\
K_{j}^{2} & \leq \min \left\{\Pi_{1}, \Pi_{2}\right\}, \\
\lambda_{i}> & \sum_{i=1}^{n}\left[\left|\check{d}_{i}-\widehat{d}_{i}\right| T_{i}+\sum_{j=1}^{m}\left|\check{a}_{j i}-\widehat{a}_{j i}\right| L_{y} R_{j}\right. \\
& \left.+\sum_{j=1}^{m}\left|\check{b}_{j i}-\widehat{b}_{j i}\right| \Gamma_{j}+\sum_{j=1}^{m}\left|\check{c}_{j i}-c_{j i}\right| \mu L_{y} R_{j}\right], \\
\lambda_{j} & >\sum_{i=1}^{n}\left[\left|\check{p}_{j}-\widehat{p}_{j}\right| R_{j}+\sum_{i=1}^{n}\left|\check{m}_{i j}-\widehat{m}_{i j}\right| L_{x} T_{i}\right. \\
& \left.+\sum_{i=1}^{n}\left|\check{n}_{i j}-\widehat{n}_{i j}\right| \Delta_{i}+\sum_{i=1}^{n}\left|\check{q}_{i j}-\widehat{q}_{i j}\right| \varepsilon L_{x} L_{i}\right],
\end{aligned}
$$

where

$$
\begin{aligned}
L_{x}= & \max \left\{\left|\alpha_{i}\right|,\left|\beta_{i}\right|\right\}, \\
L_{y}= & \max \left\{\left|\alpha_{j}\right|,\left|\beta_{j}\right|\right\}, \\
\Xi_{1}= & \check{d}_{i}-\sum_{j=1}^{m}\left(\check{a}_{j i}^{2} L_{y}^{2}+\check{b}_{j i}^{2} L_{y}^{2}+\check{c}_{j i}^{2}\right)-\frac{1}{1-d_{1}^{\prime}}-m \varepsilon L_{x}^{2} \\
& -\frac{1}{1-\sigma_{1}}, \\
\Xi_{2}= & \widehat{d}_{i}-\sum_{j=1}^{m}\left(\widehat{a}_{j i}^{2} L_{y}^{2}+\widehat{b}_{j i}^{2} L_{y}^{2}+\widehat{c}_{j i}^{2}\right)-\frac{1}{1-d_{1}^{\prime}}-m \varepsilon L_{x}^{2} \\
& -\frac{1}{1-\sigma_{1}},
\end{aligned}
$$




$$
\begin{aligned}
\Pi_{1}= & \check{p}_{j}-\sum_{i=1}^{n}\left(\check{m}_{i j}^{2} L_{x}^{2}+\check{n}_{i j}^{2} L_{x}^{2}+\check{q}_{i j}^{2}\right)-\frac{1}{1-d_{2}^{\prime}}-n \mu L_{y}^{2} \\
& -\frac{1}{1-\tau_{1}}, \\
\Pi_{2}= & \widehat{p}_{j}-\sum_{i=1}^{n}\left(\widehat{m}_{i j}^{2} L_{x}^{2}+\widehat{n}_{i j}^{2} L_{x}^{2}+\widehat{q}_{i j}^{2}\right)-\frac{1}{1-d_{2}^{\prime}}-n \mu L_{y}^{2} \\
& -\frac{1}{1-\tau_{1}} .
\end{aligned}
$$

Proof. The proof process is similar to Theorem 11. So the proof is omitted.

Remark 16. Some existing researches show the criterion for systems with differentiable delays, bounded in the in the leakage term. However, when the time-varying delays $\delta(t)$ and $\zeta(t)$ are not differentiable or their derivatives are unknown or no bounded, the criterion cannot be applicable any more. Under these circumstances, the conclusion we obtained is more valid and effective than the exciting results.

Corollary 17. Assume that Assumption 7 is satisfied; then systems (65) and (66) with constant delays in the leakage term $(\delta(t)=\delta, \zeta(t)=\zeta)$ will achieve asymptotic synchronization under the same criteria.

Remark 18. There is no extra restraint on activation functions demanding that they are bounded and the time-varying delays are mixed. Furthermore, overall consideration of our obtained results with sampled-data control schemes is shown, which can be expected to have a powerful potential application in areas like associative memory, image encryption, digital processing, and so on.

\section{Numerical Simulation}

In this section, numerical examples are presented to demonstrate the results for plausibility and validity. Based on the following parameters, consider two-dimensional MBAMNNs with mixed time-varying delays and stochastic perturbations as follows:

$$
\begin{aligned}
& d x_{i}(t)=\left[-d_{i}\left(x_{i}(t)\right) x_{i}(t)\right. \\
& +\sum_{j=1}^{2} a_{j i}\left(x_{i}(t)\right) f_{j}\left(y_{j}(t)\right)+I_{i}(t) \\
& +\sum_{j=1}^{2} b_{j i}\left(x_{i}(t-\tau(t))\right) f_{j}\left(y_{j}(t-\tau(t))\right) \\
& \left.+\sum_{j=1}^{2} c_{j i}\left(x_{i}(t)\right) \int_{t-\mu(t)}^{t} f_{j}\left(y_{j}(s)\right) d s\right] d t+\sum_{j=1}^{2} \beta_{j i} \\
& \quad \cdot\left(t, y_{j}(t), y_{j}(t-\tau(t))\right) d \omega_{j}(t),
\end{aligned}
$$

$$
\begin{aligned}
& d y_{j}(t)=\left[-p_{j}\left(y_{j}(t)\right) y_{j}(t)\right. \\
& +\sum_{i=1}^{2} m_{i j}\left(y_{j}(t)\right) g_{i}\left(x_{i}(t)\right)+I_{j}(t) \\
& +\sum_{i=1}^{2} n_{i j}\left(y_{j}(t-\sigma(t))\right) g_{i}\left(x_{i}(t-\sigma(t))\right) \\
& \left.+\sum_{i=1}^{2} q_{i j}\left(y_{j}(t)\right) \int_{t-\varepsilon(t)}^{t} g_{i}\left(x_{i}(s)\right) d s\right] d t \\
& +\sum_{i=1}^{2} \beta_{i j}\left(t, x_{i}(t), x_{i}(t-\sigma(t))\right) d \omega_{i}(t),
\end{aligned}
$$

and the corresponding response system can be defined as

$$
\begin{aligned}
& d \widehat{x}_{i}(t)=\left[-d_{i}\left(\widehat{x}_{i}(t)\right) \widehat{x}_{i}(t)\right. \\
& +\sum_{j=1}^{2} a_{j i}\left(\widehat{x}_{i}(t)\right) f_{j}\left(\hat{y}_{j}(t)\right) \\
& +\sum_{j=1}^{2} b_{j i}\left(\widehat{x}_{i}(t-\tau(t))\right) f_{j}\left(\widehat{y}_{j}(t-\tau(t))\right) \\
& +\sum_{j=1}^{2} c_{j i}\left(\widehat{x}_{i}(t)\right) \int_{t-\mu(t)}^{t} f_{j}\left(\widehat{y}_{j}(s)\right) d s+I_{i}(t) \\
& \left.+K_{i} e_{i}\left(t-d_{1}(t)\right)-\lambda_{i} \operatorname{sign}\left(e_{i}(t)\right)\right] d t \\
& +\sum_{j=1}^{2} \beta_{j i}\left(t, \hat{y}_{j}(t), \hat{y}_{j}(t-\tau(t))\right) d \omega_{j}(t), \\
& d \widehat{y}_{j}(t)=\left[-p_{j}\left(\widehat{y}_{j}(t)\right) \widehat{y}_{j}(t)\right. \\
& +\sum_{i=1}^{2} m_{i j}\left(\widehat{y}_{j}(t)\right) g_{i}\left(\widehat{x}_{i}(t)\right) \\
& +\sum_{i=1}^{2} n_{i j}\left(\widehat{y}_{j}(t-\sigma(t))\right) g_{i}\left(\widehat{x}_{i}(t-\sigma(t))\right) \\
& +\sum_{i=1}^{2} q_{i j}\left(\widehat{y}_{j}(t)\right) \int_{t-\varepsilon(t)}^{t} g_{i}\left(\widehat{x}_{i}(s)\right) d s+I_{j}(t) \\
& \left.+K_{j} e_{j}\left(t-d_{2}(t)\right)-\lambda_{j} \operatorname{sign}\left(e_{j}(t)\right)\right] d t \\
& +\sum_{i=1}^{2} \beta_{i j}\left(t, \widehat{x}_{i}(t), \widehat{x}_{i}(t-\sigma(t))\right) d \omega_{i}(t),
\end{aligned}
$$


Mathematical Problems in Engineering

17

TABLE 1: The relationship between convergence time and sampling period.

\begin{tabular}{lcccccc}
\hline Sampling period & $d=0.001$ & $d=0.01$ & $d=0.1$ & $d=1$ & $d=10$ & $d=100$ \\
\hline Convergence time & 1.616 & 1.566 & 1.515 & 3.030 & 10.510 & Unstable \\
\hline
\end{tabular}

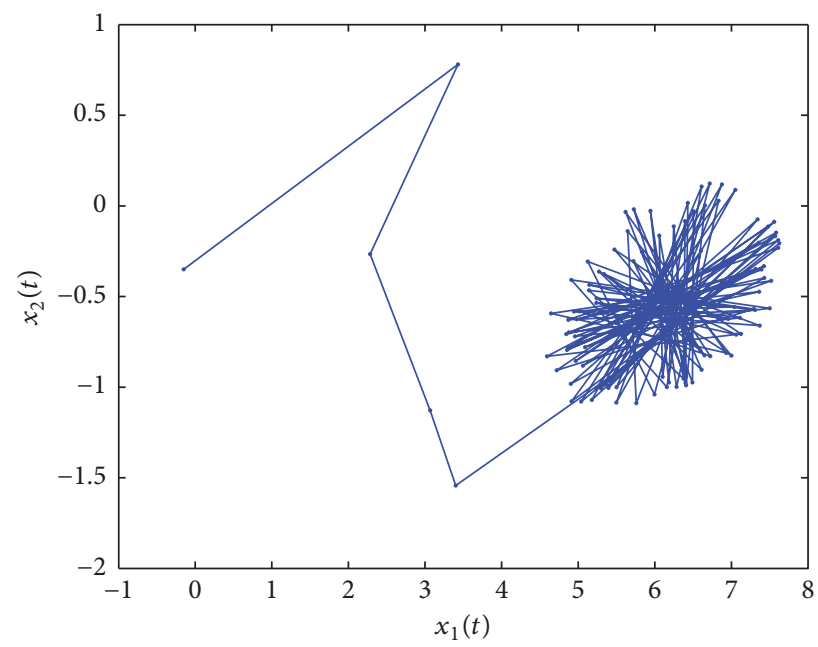

(a)

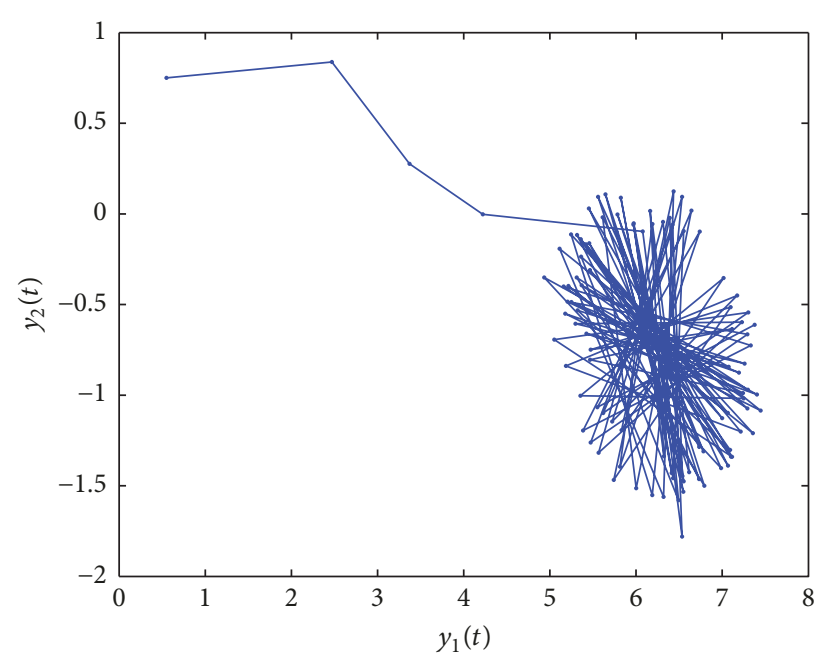

(b)

Figure 2: (a) Phase trajectories of the $x$-layer of system (3). (b) Phase trajectories of the $y$-layer of system (3).

where

$$
\begin{aligned}
& d_{1}\left(x_{1}(t)\right)= \begin{cases}-1.1, & \left|x_{1}(t)\right| \leq 1, \\
-1.2, & \left|x_{1}(t)\right|>1,\end{cases} \\
& a_{11}\left(x_{1}(t)\right)= \begin{cases}1.3, & \left|x_{1}(t)\right| \leq 1, \\
1.4, & \left|x_{1}(t)\right|>1,\end{cases} \\
& c_{21}\left(x_{1}(t)\right)= \begin{cases}0.18, & \left|x_{1}(t)\right| \leq 1, \\
-0.13, & \left|x_{1}(t)\right|>1,\end{cases} \\
& d_{2}\left(x_{2}(t)\right)= \begin{cases}-1, & \left|x_{2}(t)\right| \leq 1, \\
-1.1, & \left|x_{2}(t)\right|>1,\end{cases} \\
& p_{1}\left(y_{1}(t)\right)= \begin{cases}-1.2, & \left|y_{1}(t)\right| \leq 2, \\
-1.1, & \left|y_{1}(t)\right|>2,\end{cases} \\
& p_{2}\left(y_{2}(t)\right)= \begin{cases}-0.9, & \left|y_{2}(t)\right| \leq 2, \\
-1.3, & \left|y_{2}(t)\right|>2,\end{cases} \\
& q_{11}\left(y_{1}(t)\right)= \begin{cases}1.4, & \left|y_{1}(t)\right| \leq 2, \\
1.33, & \left|y_{1}(t)\right|>2,\end{cases}
\end{aligned}
$$

$$
\begin{aligned}
& a_{12}\left(x_{2}(t)\right)= \begin{cases}1.8, & \left|x_{2}(t)\right| \leq 1, \\
1.9, & \left|x_{2}(t)\right|>1,\end{cases} \\
& a_{22}\left(x_{2}(t)\right)= \begin{cases}0.48, & \left|x_{1}(t)\right| \leq 1, \\
1.51, & \left|x_{1}(t)\right|>1,\end{cases} \\
& m_{12}\left(y_{2}(t)\right)= \begin{cases}1.75, & \left|y_{2}(t)\right| \leq 2, \\
1.79, & \left|y_{2}(t)\right|>2,\end{cases} \\
& m_{22}\left(y_{2}(t)\right)= \begin{cases}0.51, & \left|y_{2}(t)\right| \leq 2, \\
1.48, & \left|y_{2}(t)\right|>2,\end{cases} \\
& c_{12}\left(x_{2}(t)\right)= \begin{cases}0.21, & \left|x_{2}(t)\right| \leq 1, \\
0.32, & \left|x_{2}(t)\right|>1,\end{cases} \\
& a_{21}\left(x_{1}(t)\right)= \begin{cases}7.2, & \left|x_{1}(t)\right| \leq 1, \\
5.1, & \left|x_{1}(t)\right|>1,\end{cases} \\
& q_{11}\left(y_{1}(t)\right)= \begin{cases}-0.48, & \left|y_{1}(t)\right| \leq 2, \\
-0.24, & \left|y_{1}(t)\right|>2,\end{cases} \\
& m_{21}\left(y_{1}(t)\right)= \begin{cases}6.83, & \left|x_{2}(t)\right| \leq 2, \\
4.96, & \left|y_{1}(t)\right|>2,\end{cases}
\end{aligned}
$$



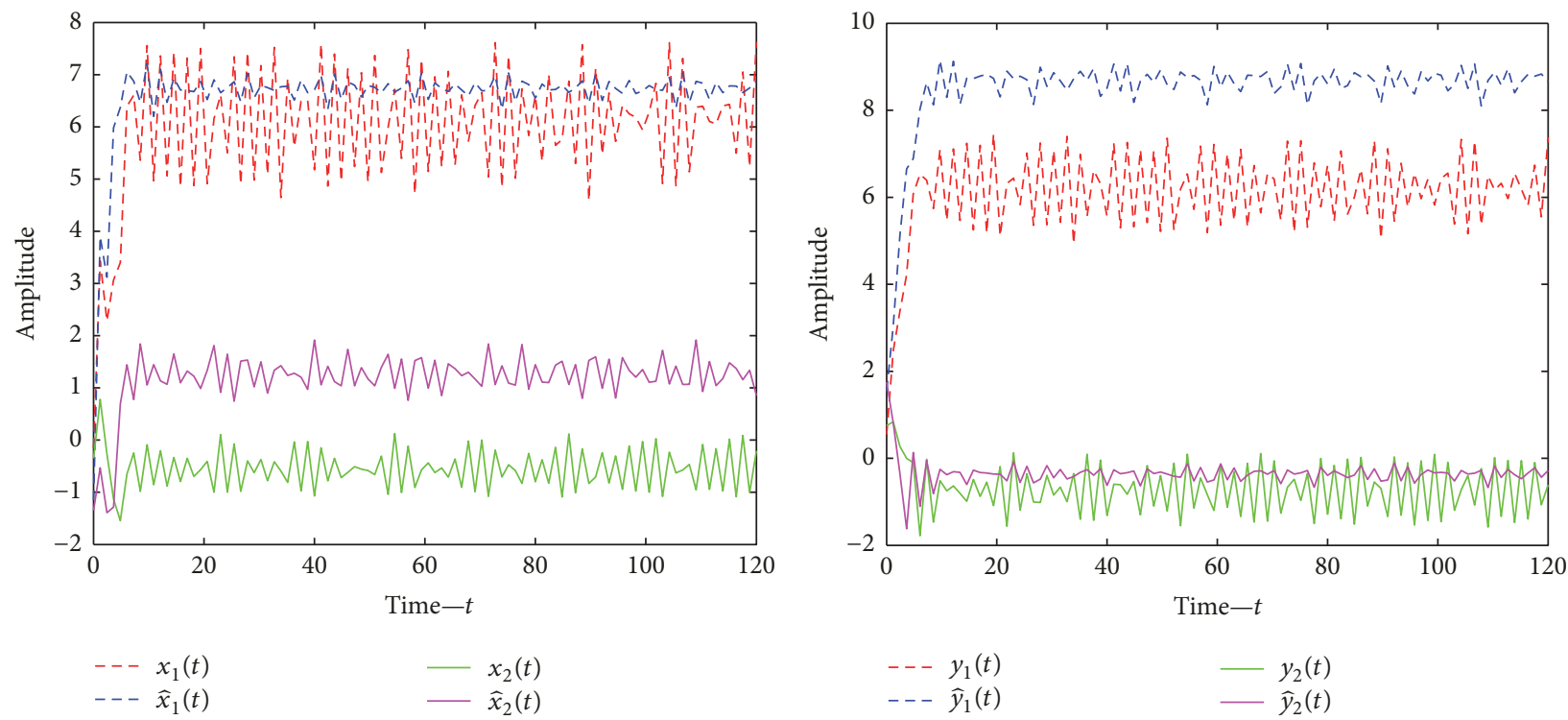

(a)

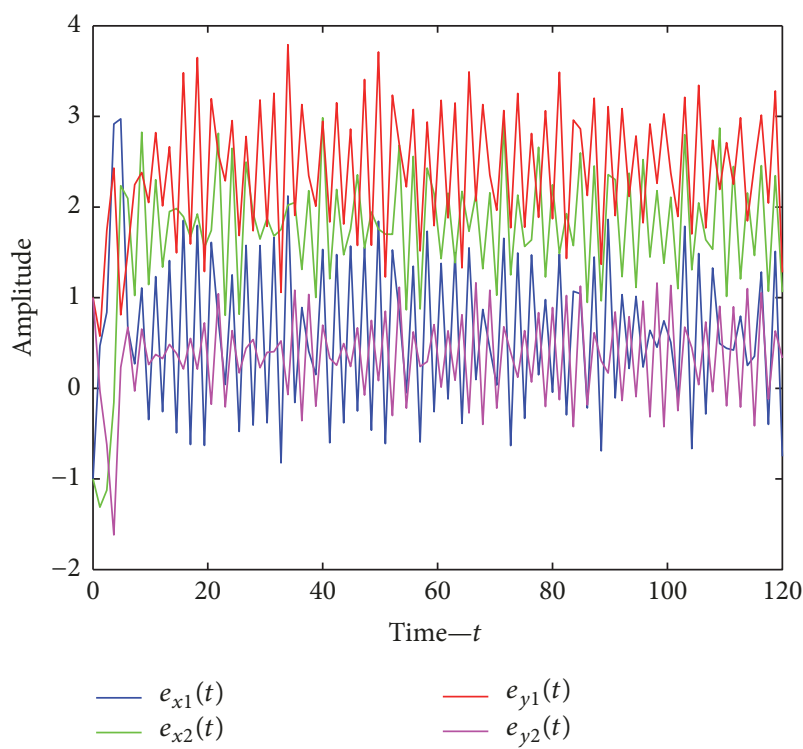

(c)

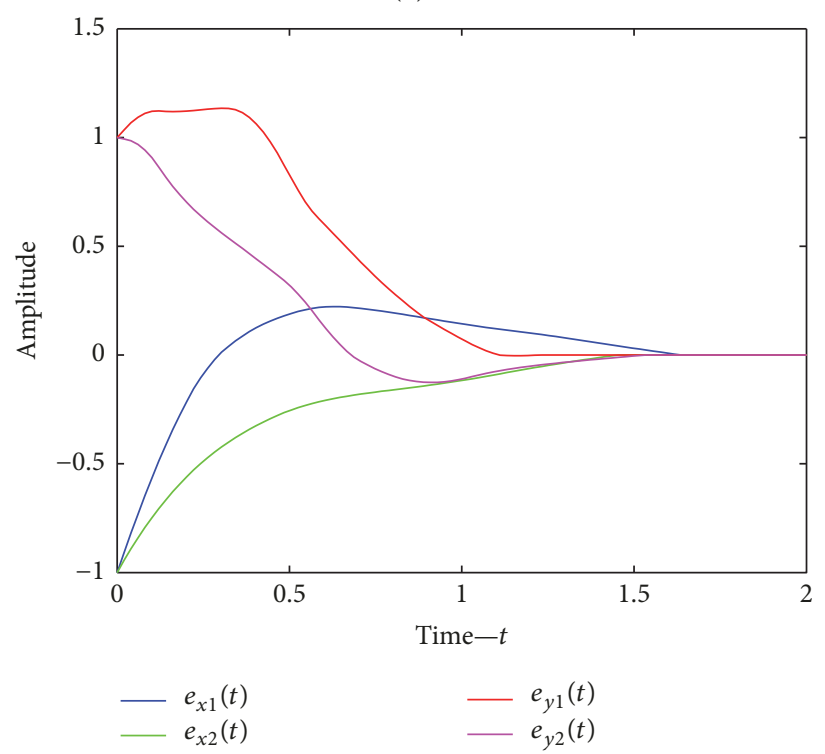

(d)

Figure 3: (a) and (b) show the time response curves of drive-response systems (69) and (70); (c) and (d) show the curves of error systems without or with delay-dependent controller (14), respectively.

$$
\begin{aligned}
& c_{11}\left(x_{1}(t)\right)= \begin{cases}0.56, & \left|x_{1}(t)\right| \leq 1, \\
-0.4, & \left|x_{1}(t)\right|>1,\end{cases} \\
& b_{22}\left(x_{2}(t-\tau(t))\right)= \begin{cases}-1.32, & \left|x_{2}(t-\tau(t))\right| \leq 1, \\
-1.43, & \left|x_{2}(t-\tau(t))\right|>1,\end{cases} \\
& q_{12}\left(y_{2}(t)\right)= \begin{cases}0.49, & \left|y_{2}(t)\right| \leq 2, \\
0.42, & \left|y_{2}(t)\right|>2,\end{cases} \\
& n_{11}\left(y_{1}(t-\sigma(t))\right)= \begin{cases}-1.51, & \left|y_{1}(t-\sigma(t))\right| \leq 2, \\
-1.20, & \left|y_{1}(t-\sigma(t))\right|>2,\end{cases} \\
& q_{22}\left(y_{2}(t)\right)= \begin{cases}-0.31, & \left|y_{2}(t)\right| \leq 2, \\
-0.42, & \left|y_{2}(t)\right|>2 .\end{cases} \\
& n_{21}\left(y_{1}(t-\sigma(t))\right)= \begin{cases}0.81, & \left|y_{1}(t-\sigma(t))\right| \leq 2, \\
0.9, & \left|y_{1}(t-\sigma(t))\right|>2,\end{cases} \\
& b_{12}\left(x_{2}(t-\tau(t))\right)= \begin{cases}0.76, & \left|x_{2}(t-\tau(t))\right| \leq 1, \\
0.98, & \left|x_{2}(t-\tau(t))\right|>1,\end{cases} \\
& b_{11}\left(x_{1}(t-\tau(t))\right)= \begin{cases}-1.48, & \left|x_{1}(t-\tau(t))\right| \leq 1, \\
-1.19, & \left|x_{1}(t-\tau(t))\right|>1,\end{cases}
\end{aligned}
$$




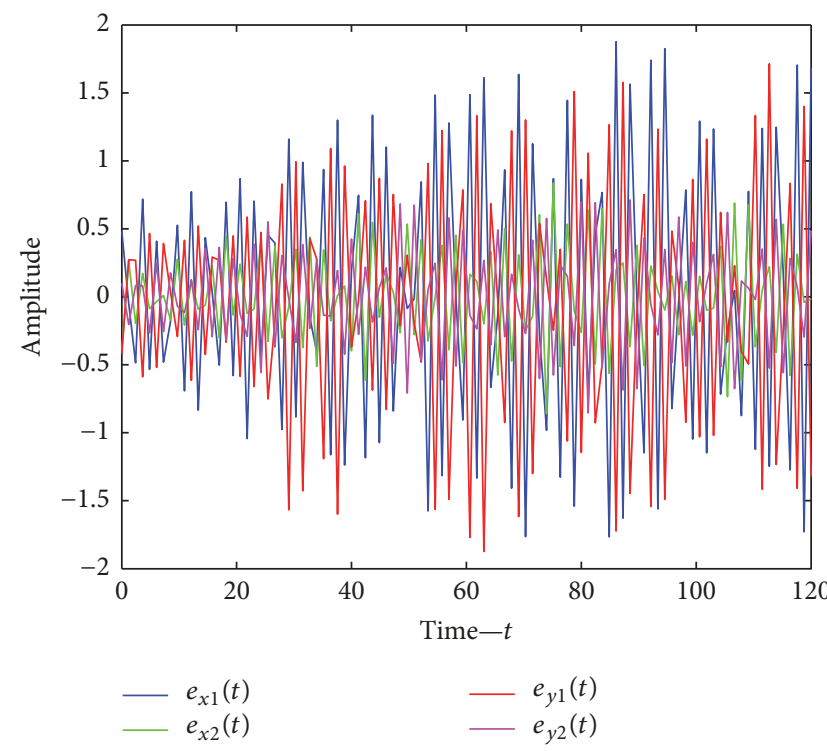

(a)

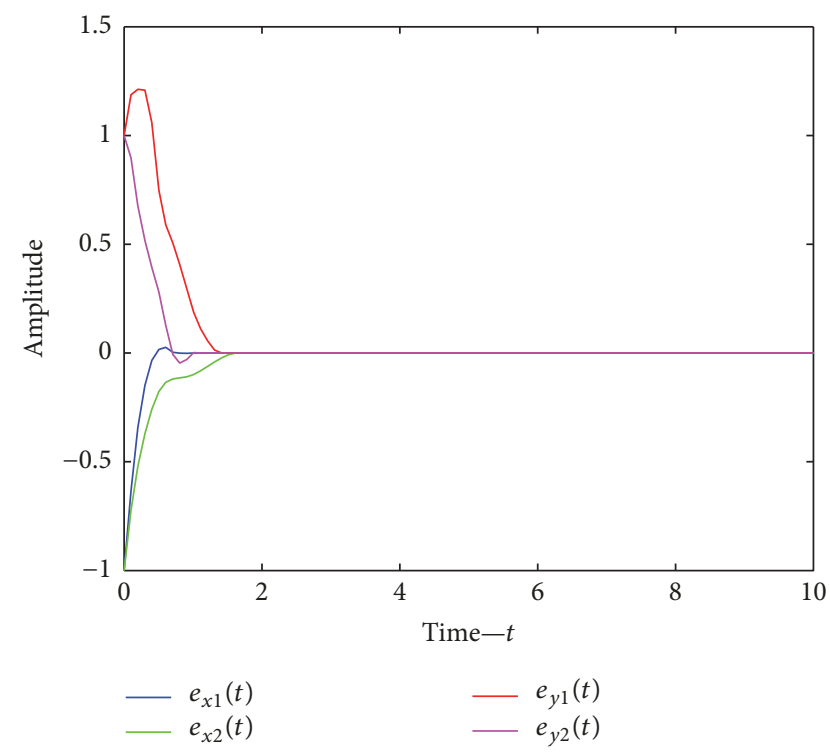

(b)

FIGURE 4: (a) The synchronization error of drive-response systems (69) and (70) without perturbations and control; (b) the synchronization error of drive-response systems (69) and (70) without perturbations but under control.

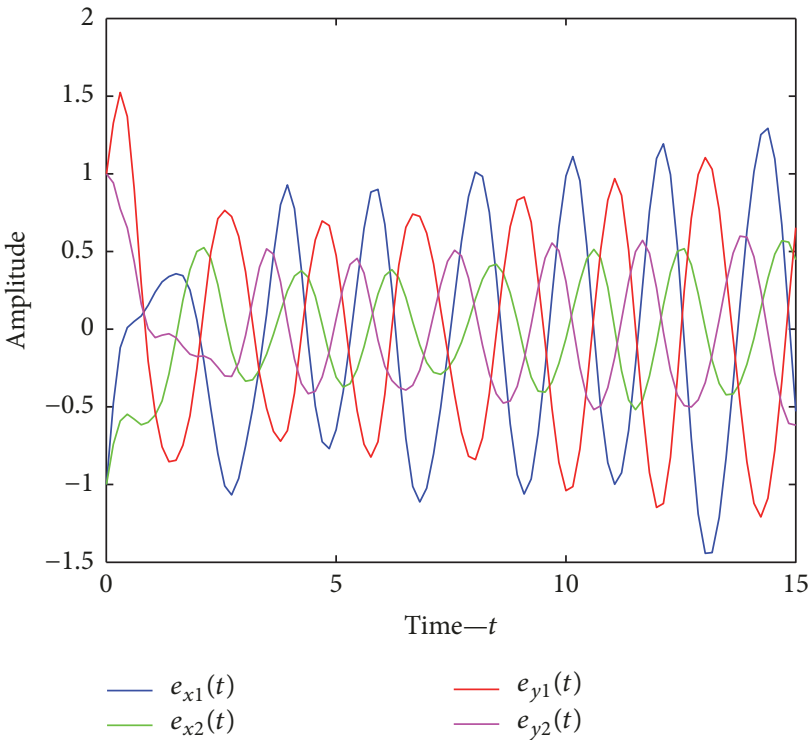

(a)

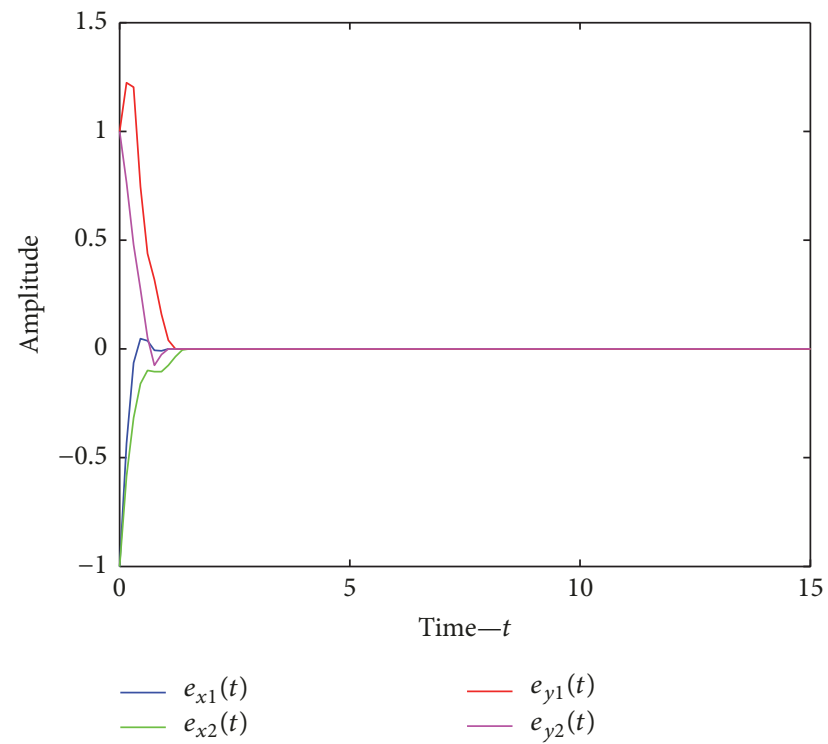

(b)

Figure 5: (a) The synchronization error of drive-response systems (65) and (66) without control; (b) the synchronization error of driveresponse systems (65) and (66) under control.

$$
\begin{aligned}
& b_{21}\left(x_{1}(t-\tau(t))\right)= \begin{cases}0.88, & \left|x_{1}(t-\tau(t))\right| \leq 1, \\
0.75, & \left|x_{1}(t-\tau(t))\right|>1,\end{cases} \\
& n_{12}\left(y_{2}(t-\sigma(t))\right)= \begin{cases}0.83, & \left|y_{2}(t-\sigma(t))\right| \leq 2, \\
0.99, & \left|y_{2}(t-\sigma(t))\right|>2,\end{cases} \\
& n_{22}\left(y_{2}(t-\sigma(t))\right)= \begin{cases}-1.42, & \left|y_{2}(t-\sigma(t))\right| \leq 2, \\
-1.52, & \left|y_{2}(t-\sigma(t))\right|>2 .\end{cases}
\end{aligned}
$$

Taking the activation function as $f_{1}(\cdot)=g_{1}(\cdot)=\sin (|\cdot|)$ and $f_{2}(\cdot)=g_{2}(\cdot)=\tanh (|\cdot|)$, we have $\tau(t)=\sigma(t)=0.4-$ $0.1 \sin t, \mu(t)=\varepsilon(t)=0.5+0.5 \cos t, \delta(t)=\zeta(t)=0.15 t$. The sampling period is taken as $d_{1}(t)=d_{2}(t)=0.1 \sin t$.

We choose the initial values of the state variables as $\left[x_{1}(t), x_{2}(t)\right]=[-0.15,-0.35],\left[\widehat{x}_{1}(t), \widehat{x}_{2}(t)\right]=[-1.15,-1.35]$, $\left[y_{1}(t), y_{2}(t)\right]=[0.55,0.75]$, and $\left[\widehat{y}_{1}(t), \widehat{y}_{2}(t)\right]=[1.55,1.75]$. We also define the external input $\left[I_{i}(t), I_{j}(t)\right]^{T}=[0,0]^{T}$.

We make $L_{x}=L_{y}=0.001, J_{1}=J_{2}=H_{1}=H_{2}=0.00001$; thus according to the Theorem 11, we calculate $K_{i}=-0.2$, $K_{j}=-1, \lambda_{i}=\lambda_{j}=-0.2$. 


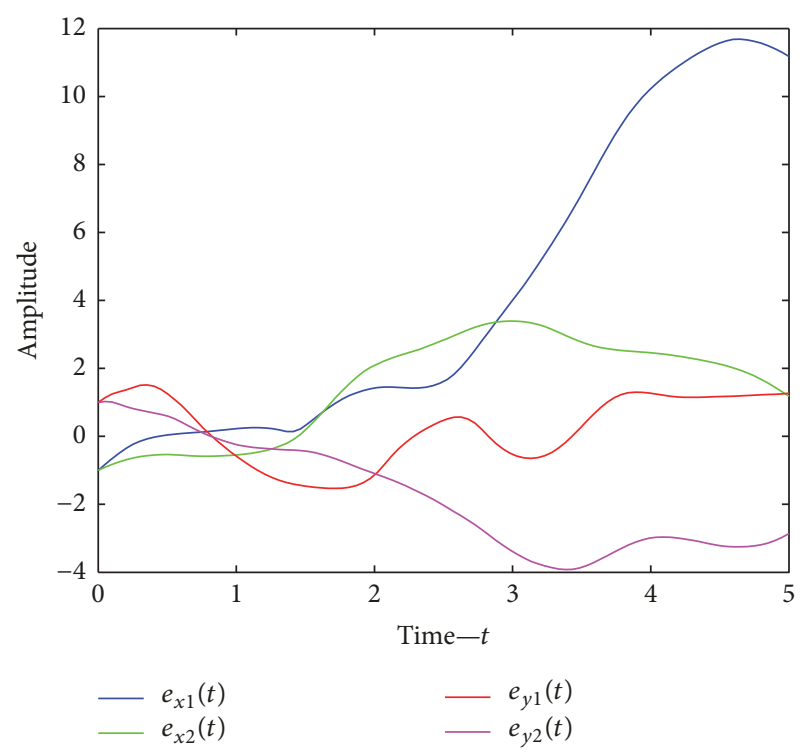

(a)

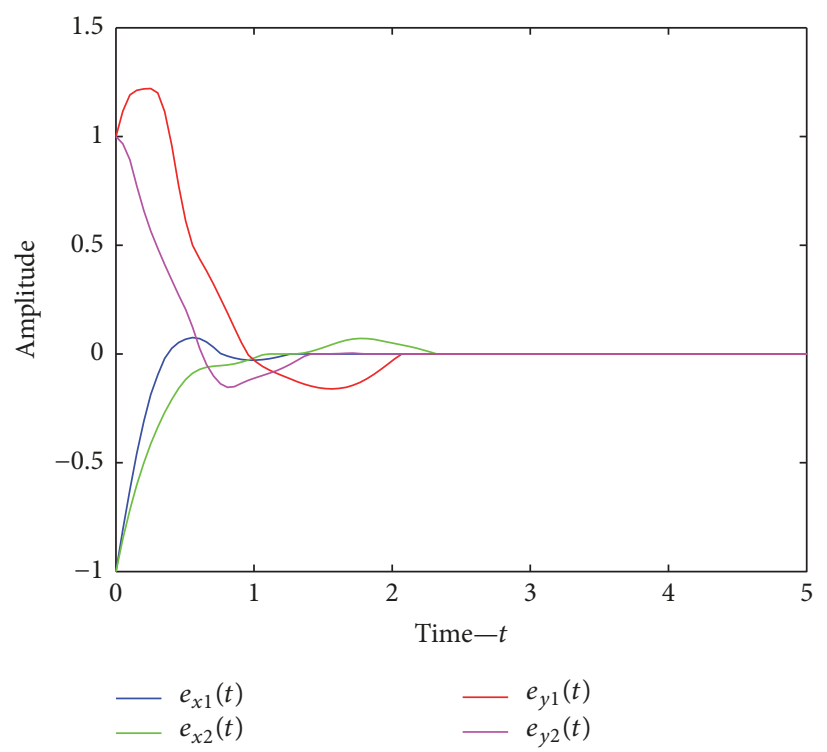

(b)

FIGURE 6: (a) The synchronization error of drive-response systems (65) and (66) with constant leakage delays but without control; (b) the synchronization error of drive-response systems (65) and (66) with constant leakage delays and control.

The Brownian motion satisfies $E \omega(t)=0, D \omega(t)=1$.

$$
\begin{aligned}
& \beta_{\mathbf{j i}}(\mathbf{t}), \mathbf{e}_{\mathbf{j}}(\mathbf{t}), \mathbf{e}_{\mathbf{j}}(\mathbf{t}-\tau(\mathbf{t}))=\operatorname{diag}\left\{0.4 e_{j}(t)\right. \\
& \left.\quad+0.3 e_{j}(t-\tau(t)),-0.5 e_{j}(t)+0.2 e_{j}(t-\tau(t))\right\}, \\
& \beta_{\mathbf{i j}}(\mathbf{t}), \mathbf{e}_{\mathbf{i}}(\mathbf{t}), \mathbf{e}_{\mathbf{i}}(\mathbf{t}-\sigma(\mathbf{t}))=\operatorname{diag}\left\{0.4 e_{i}(t)\right. \\
& \left.\quad+0.3 e_{i}(t-\sigma(t)),-0.5 e_{i}(t)+0.2 e_{i}(t-\sigma(t))\right\} .
\end{aligned}
$$

The dynamic behaviors of drive-response systems are given in Figure 2, and the following simulations are conducted on the basis of this situation. In order to verify Theorem 11, we take systems (69) and (70) as example. Figure 3 shows the state trajectories of such systems without control; then it illustrates the synchronization errors correlation between the drive-response systems without and under control. From the above illustration, we conclude that systems (69) and (70) will achieve globally asymptotic synchronization with the help of the proposed controller (14).

Based on Theorem 11, we indicate the considered systems without perturbations. Under these circumstances, define the errors as $d e_{i}(t)=d \widehat{x}_{i}(t)-d x_{i}(t)$ and $d e_{j}(t)=d \widehat{y}_{j}(t)-$ $d y_{j}(t)$; the error states without control and under control are depicted in Figure 4. The comparison between (a) and (b) provides the clearest significant role of the controller played in the synchronization control.

According to Corollary 15, for given time-varying delays $\delta(t)$ and $\zeta(t)$, systems (65) and (66) are globally asymptotically synchronized under controller (14). In this simulation, we take $\delta(t)=\zeta(t)=0.15 t$, and Figure 5 depicts the trajectories of error states. In order to testify Corollary 17, we make $\delta=\zeta=0.15$ in the leakage terms; Figure 6 illustrates the error states of the proposed systems. From the comparison results we conclude the sampled-data control inputs which contribute to the effective chaos synchronization.

Based on the model of Corollary 15, we choose the different sampling periods to verify the relationship between sampling period and the convergence time of error systems. We set $d_{1}(t)=d_{2}(t)=0.001 \sin t, d_{1}(t)=d_{2}(t)=0.01 \sin t$, $d_{1}(t)=d_{2}(t)=0.1 \sin t, d_{1}(t)=d_{2}(t)=1 \sin t, d_{1}(t)=$ $d_{2}(t)=10 \sin t, d_{1}(t)=d_{2}(t)=100 \sin t$, respectively. Figure 7 depicts the different error convergence corresponding to the different sampling period. Table 1 gives the specific convergence times corresponding to the different sampling periods. We find that the larger the sampling period we take, the worse the effect on the error convergence.

The error system becomes unstable when we choose sampling period $d_{1}=d_{2}=100$. Under these circumstances, it means the sampled-data controller with the feedback gains $K_{i}$ and $K_{j}$ is not enough to guarantee the stability of the error systems. For comparing and analyzing the influence of the sampling period relationship, we make the state feedback controller with the same feedback gains. Figure 7 depicts the trajectories of error states. From Figure 8 we detect that the error system becomes stable at 11.921. So we conclude that when we take the sampled-data controller, the effect on error convergence is not only relevant to the feedback gains but also related to the sampling period. Table 1 illustrates that the best sampling period for our simulations is $d_{1}(t)=d_{2}(t)=$ $0.01 \sin t$.

Remark 19. Comparing with the exciting researches on MBAMNNs, we investigate the relationship between the sampling period and convergence time. It can be found that even the longer sampling period will cause the lower communication channel occupation, less packet transmission, and little actuation of the controller; it also sacrifices the accuracy 

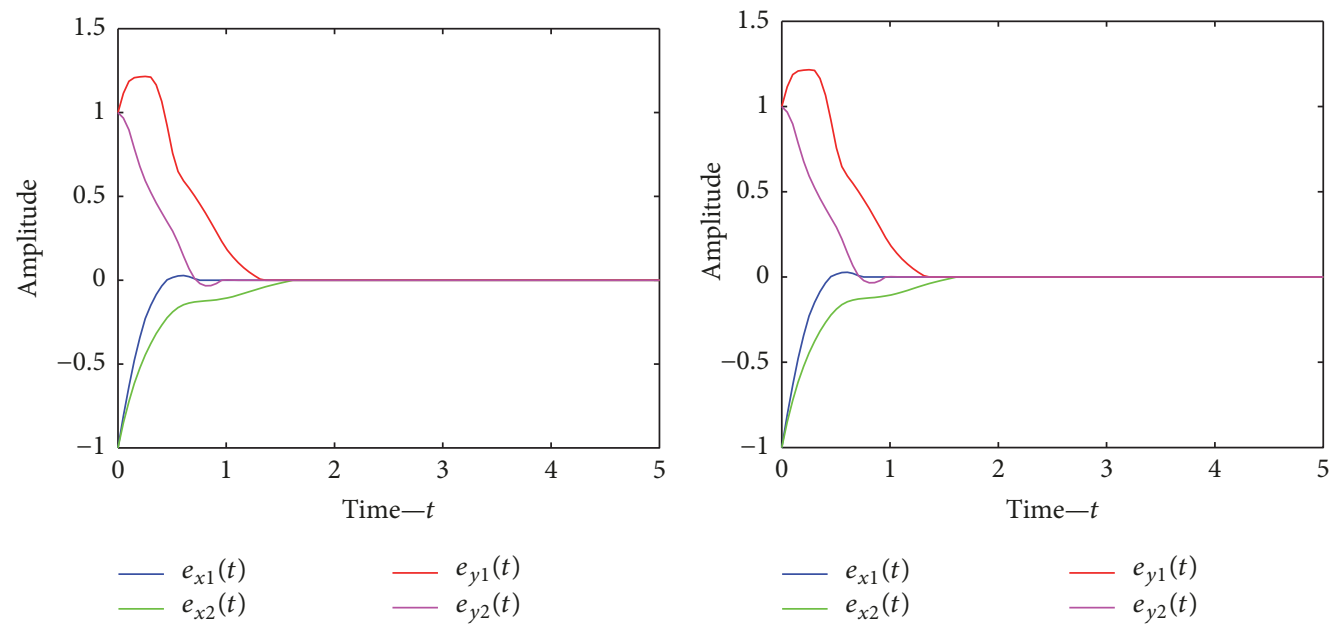

(a)

(b)
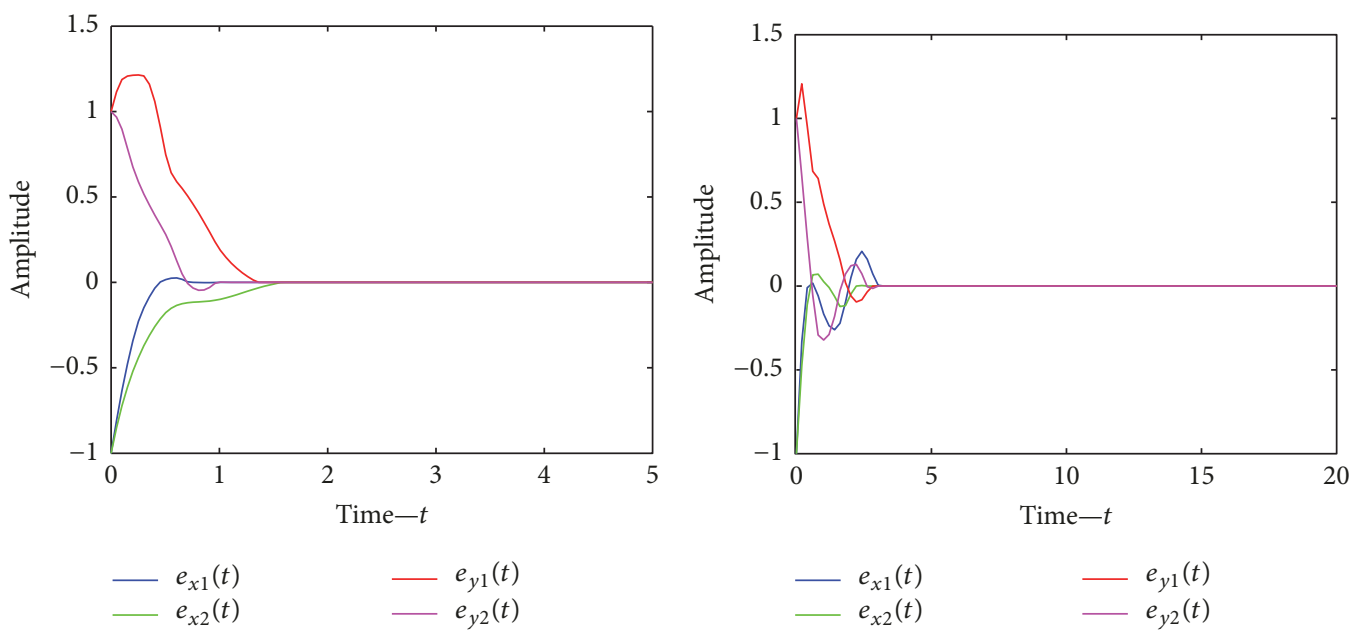

(c)

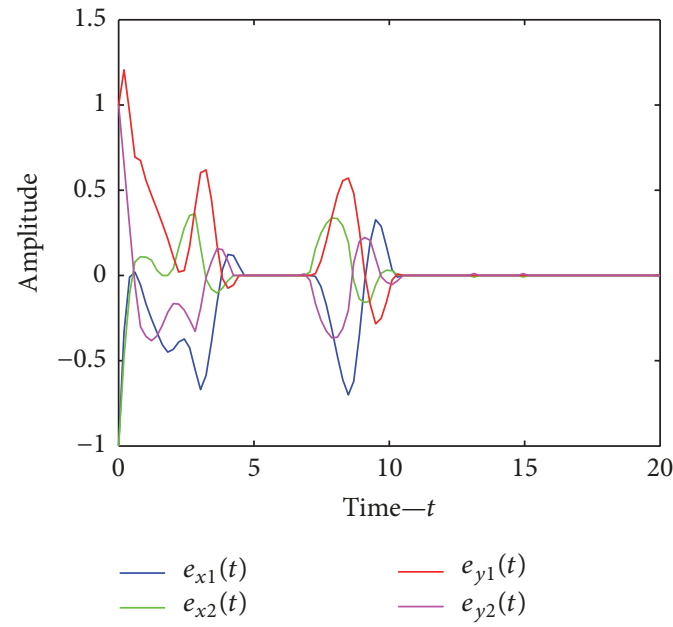

(e)

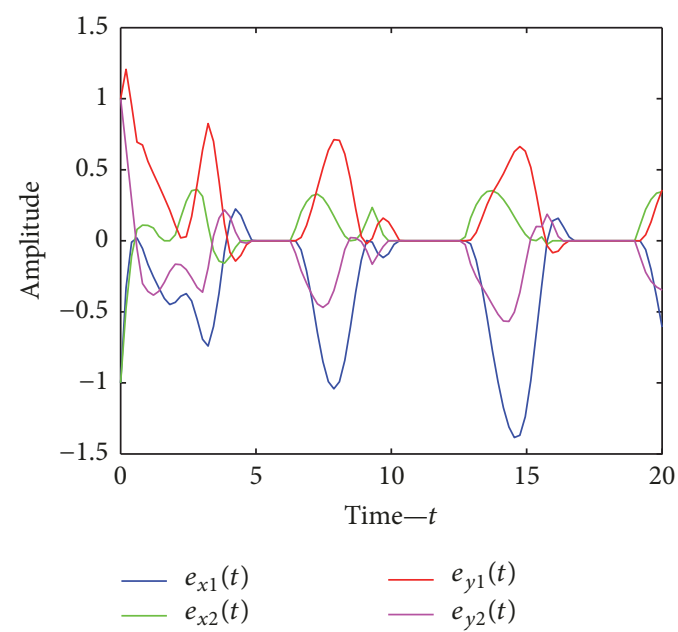

(f)

FIGURE 7: (a) The synchronization error of sampled period $d_{1}(t)=d_{2}(t)=0.001 \sin t$; (b) $d_{1}(t)=d_{2}(t)=0.01 \sin t$; (c) $d_{1}(t)=d_{2}(t)=$ $0.1 \sin t ;(\mathrm{d}) d_{1}(t)=d_{2}(t)=1 \sin t ;(\mathrm{e}) d_{1}(t)=d_{2}(t)=10 \sin t ;(\mathrm{f}) d_{1}(t)=d_{2}(t)=100 \sin t$. 


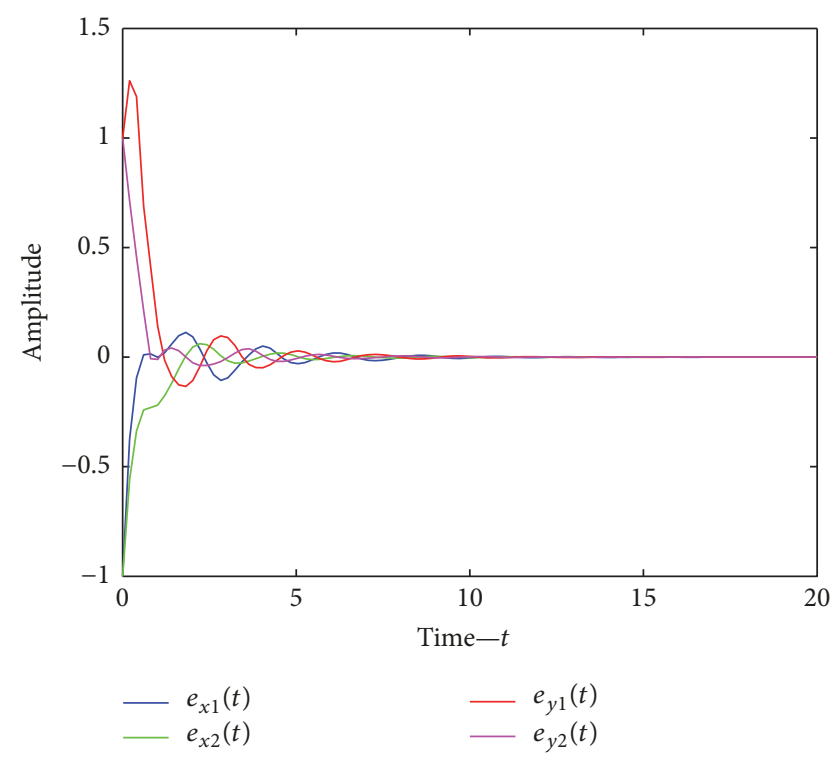

FIGURE 8: The synchronization error of drive-response systems (69) and (70) without perturbations but under normal feedback controller.

of controller at the same time. Thus the suitable sampling period has important significance to the stable of error systems. So our conclusion is greatly helpful to some potential future research topics including synchronization or stability on sampled-data synchronization strategy to NNs.

\section{Conclusion}

In this paper, a synchronization problem has been investigated for MBAMNNs with stochastic perturbations and mixed time-varying delays. The dynamics of the synaptic weights between the drive and response systems were considered and analyzed rather than treating them as consonants. Firstly, both various mixed time-varying delays and stochastic perturbations are considered in this paper, which include no-delay, finite distributed time-varying delays, discrete time-varying delays, and leakage time-varying delays. Secondly, we have proposed a sampled-data synchronization strategy for each node of the MBAMNNs. By utilizing the sign function and the definition of asymptotic stability, a suitable nonlinear state feedback sampled-data controller is designed. In addition, we verify the sampling period effect on the convergence of error system according to simulations. By utilizing the Lyapunov functional method, stochastic analysis theory, and inequality techniques, some sufficient conditions are derived to guarantee synchronization of the MBAMNNs model. Simulation examples have been presented to validate the theoretical results.

\section{Conflicts of Interest}

The authors declare that there are no conflicts of interest regarding the publication of this paper.

\section{Authors' Contributions}

Weiping Wang and Xiong Luo contributed equally to this work.

\section{Acknowledgments}

This work was supported by the National Key Research and Development Program of China under Grant 2017YFB0702300, the State Scholarship Fund of China Scholarship Council (CSC), the National Natural Science Foundation of China under Grants 61603032 and 61174103, the Fundamental Research Funds for the Central Universities under Grant 06500025, the National Key Technologies R\&D Program of China under Grant 2015BAK38B01, and the University of Science and Technology Beijing-National Taipei University of Technology Joint Research Program under Grant TW201705.

\section{References}

[1] X. Luo, Y. Xu, W. Wang et al., "Towards enhancing stacked extreme learning machine with sparse autoencoder by correntropy," Journal of The Franklin Institute, vol. 355, no. 4, pp. 19451966, 2018.

[2] X. Luo, J. Deng, J. Liu, W. Wang, X. Ban, and J. Wang, "A quantized kernel least mean square scheme with entropy-guided learning for intelligent data analysis," China Communications, vol. 14, no. 7, pp. 127-136, 2017.

[3] B. Kosko, "Bidirectional associative memories," IEEE Transactions on Systems, Man, and Cybernetics, vol. 18, no. 1, pp. 49-60, 1988.

[4] Z. Guo, J. Wang, and Z. Yan, "Attractivity analysis of memristorbased cellular neural networks with time-varying delays," IEEE Transactions on Neural Networks and Learning Systems, vol. 25, no. 4, pp. 704-717, 2014.

[5] L. O. Chua, "Memristor-the missing circuit element," IEEE Transactions on Circuit Theory, vol. 18, no. 5, pp. 507-519, 1971.

[6] D. Liu, S. Zhu, and K. Sun, "New results for exponential stability of complex-valued memristive neural networks with variable delays," Neurocomputing, pp. 758-767, 2017.

[7] Z. Guo, J. Wang, and Z. Yan, "Passivity and passification of memristor-based recurrent neural networks with time-varying delays," IEEE Transactions on Neural Networks and Learning Systems, vol. 25, no. 11, pp. 2099-2109, 2014.

[8] J. J. Chen, Z. G. Zeng, and P. Jiang, "Global exponential almost periodicity of a delayed memristor-based neural networks," Neural Networks the Official Journal of the International Neural Network Society, vol. 60, pp. 33-43, 2014.

[9] H. Wu, R. Li, X. Zhang, and R. Yao, "Adaptive finite-time complete periodic synchronization of memristive neural networks with time delays," Neural Processing Letters, vol. 42, no. 3, pp. 563-583, 2015.

[10] Y. Xu, X. Luo, W. Wang, and W. Zhao, "Efficient DV-HOP localization forwireless cyber-physical social sensing system: a correntropy-based neural network learning scheme," Sensors, vol. 17, no. 1, article 135, 2017.

[11] S. Ding and Z. Wang, "Stochastic exponential synchronization control of mem-ristive neural networks with multiple timevarying delays," Elsevier Science Publishers B. V, vol. 162, pp. 1625, 2015. 
[12] H. Zhao, L. Li, H. Peng, J. Kurths, J. Xiao, and Y. Yang, "Finitetime robust synchronization of memrisive neural network with perturbation," Neural Processing Letters, pp. 1-25, 2015.

[13] J. Xiao, S. Zhong, and Y. Li, "Improved passivity criteria for memristive neural networks with interval multiple timevarying delays," Elsevier Science Publishers B. V, vol. 171, pp. 1414-1430, 2016.

[14] M. Yu, W. Wang, X. Luo, L. Liu, and M. Yuan, "Exponential antisynchronization control of stochastic memristive neural networks with mixed time-varying delays based on novel delaydependent or delay-independent adaptive controller," Mathematical Problems in Engineering, Article ID 8314757, 2017.

[15] G. D. Zhang and Y. Shen, "Exponential synchronization of delayed memristor-based chaotic neural networks via periodically intermittent control," Neural Networks, vol. 55, pp. 1-10, 2014.

[16] A. Chandrasekar, R. Rakkiyappan, J. Cao, and S. Lakshmanan, "Synchronization of memristor-based recurrent neural networks with two delay components based on second-order reciprocally convex approach," Neural Networks, vol. 57, pp. 7993, 2014.

[17] L. Wang and Y. Shen, "Design of controller on synchronization of memristor-based neural networks with time-varying delays," Neurocomputing, vol. 147, no. 1, pp. 372-379, 2015.

[18] X. Yang, J. Cao, and W. Yu, "Exponential synchronization of memristive Cohen-Grossberg neural networks with mixed delays," Cognitive Neurodynamics, vol. 8, no. 3, pp. 239-249, 2014.

[19] H. Wu, R. Li, and H. Wei, "Synchronization of a class of memristive neural networks with time delays via sampleddata control," International Journal of Machine Learning and Cybernetics, vol. 6, pp. 365-373, 2015.

[20] X. Han, H. Wu, and B. Fang, "Adaptive exponential synchronization of memristive neural networks with mixed timevarying delays," Neurocomputing, vol. 201, pp. 40-50, 2016.

[21] L. Wang, Y. Shen, and Q. Yin, "Adaptive synchronization of memristor-based neural networks with time-varying delays," Neurocomputing, vol. 26, 2015.

[22] P. Jiang, Z. Zeng, and J. Chen, "On the periodic dynamics of memristor-based neural networks with time-varying delays," Neurocomputing, vol. 279, pp. 358-373, 2014.

[23] J. Xiao, S. Zhong, Y. Li, and F. Xu, "Finite-time Mittag-Leffler synchronization of fractional-order memristive BAM neural networks with time delays," Neurocomputing, vol. 219, pp. 431439, 2017.

[24] G. D. Zhang and Y. Shen, "New algebraic criteria for synchronization stability of chaotic memristive neural networks with time-varying delays," IEEE Transactions on Neural Networks and Learning Systems, vol. 24, no. 10, pp. 1701-1707, 2013.

[25] M. Jiang, S. Wang, J. Mei, and Y. Shen, "Finite-time synchronization control of a class of memristor-based recurrent neural networks," Neural Networks, vol. 63, pp. 133-140, 2015.

[26] K. Mathiyalagan, J. H. Park, and R. Sakthivel, "Synchronization for delayed memristive BAM neural networks using impulsive control with random nonlinearities," Applied Mathematics and Computation, vol. 259, pp. 967-979, 2015.

[27] R. Sakthivel, R. Anbuvithya, K. Mathiyalagan, Y.-K. Ma, and P. Prakash, "Reliable anti-synchronization conditions for BAM memristive neural networks with different memductance functions," Applied Mathematics and Computation, vol. 275, pp. 213228, 2016.
[28] R. Anbuvithya, K. Mathiyalagan, R. Sakthivel, and P. Prakash, "Non-fragile synchronization of memristive BAM networks with random feedback gain fluctuations," Communications in Nonlinear Science and Numerical Simulation, vol. 29, no. 1-3, pp. 427-440, 2015.

[29] M. Zarefard and S. Effati, "Adaptive synchronization between two non-identical BAM neural networks with unknown parameters and time-varying delays," International Journal of Control, Automation, and Systems, vol. 15, no. 4, pp. 1877-1887, 2017.

[30] S. Ding, Z. Wang, N. Rong, and H. Zhang, "Exponential stabilization of memristive neural networks via saturating sampleddata control," IEEE Transactions on Cybernetics, vol. 99, no. 10, pp. 1-3, 2017.

[31] K. Astrom and B. Wittenmark, Computer-Controlled Systems: Theory and Design, Dover, New York, NY, USA, 2011.

[32] G. Chao, W. Hong, Y. Liu, and J. Park, "Improved stabilization criteria for fuzzy systems under variable sampling," Journal of The Franklin Institute, vol. 354, no. 14, pp. 5839-5853, 2017.

[33] G. Chao, B. Wang, X. Wei, and Y. Liu, "Exponential synchronization of a class of neural networks with sampled-data control," Applied Mathematics and Computation, vol. 315, pp. 150-161, 2017.

[34] M. Zheng, L. Li, H. Peng, J. Xiao, Y. Yang, and H. Zhao, "Finite-time projective synchronization of memristor-based delay fractional-order neural networks," Nonlinear Dynamics, vol. 89, no. 4, pp. 2641-2655, 2017.

[35] W. Wang, M. Yu, X. Luo, L. Liu, M. Yuan, and W. Zhao, "Synchronization of memristive BAM neural networks with leakage delay and additive time-varying delay components via sampled-data control," Chaos, Solitons and Fractals, vol. 104, pp. 84-97, 2017.

[36] M. Yuan, X. Luo, W. Wang, L. Li, and H. Peng, "Pinning synchronization of coupled memristive recurrent neural networks with mixed time-varying delays and perturbations," Neural Processing Letters, 2018.

[37] H. Bao, J. H. Park, and J. Cao, "Exponential synchronization of coupled stochastic memristor-based neural networks with time-varying probabilistic delay coupling and impulsive delay," IEEE Transactions on Neural Networks and Learning Systems, vol. 27, no. 1, pp. 190-201, 2016.

[38] F. Wang and M. Liu, "Global exponential stability of high-order bidirectional associative memory (BAM) neural networks with time delays in leakage terms," Neurocomputing, vol. 177, pp. 515528, 2016.

[39] H. Cai and L. Huang, "Functional differential inclusions and dynamic behaviors for memristor-based BAM neural networks with time-varying delays," Communications in Nonlinear Science and Numerical Simulation, vol. 19, no. 5, pp. 1279-1300, 2014.

[40] C. Hua, C. Ge, and X. Guan, "Synchronization of chaotic Lur'e systems with time delays using sampled-data control," IEEE Transactions on Neural Networks and Learning Systems, vol. 26, no. 6, pp. 1214-1221, 2015.

[41] H. Wu, R. Li, and H. Wei, "Synchronization of a class of memristive neural networks with time delays via sampled-data control," vol. 6, pp. 365-373, 2015.

[42] Y. Shi and P. Zhu, "Finite-time synchronization of stochastic memristor-based delayed neural networks," Neural Computing and Applications, pp. 1-9, 2016.

[43] S. Wen, Z. Zeng, T. Huang, Q. Meng, and W. Yao, "Lag synchronization of switched neural networks via neural activation function and applications in image encryption," IEEE 
Transactions on Neural Networks and Learning Systems, vol. 26, no. 7, pp. 1493-1502, 2015.

[44] M. Syed Ali, R. Saravanakumar, and J. Cao, "New passivity criteria for memristor-based neutral-type stochastic BAM neural networks with mixed time-varying delays," Neurocomputing, vol. 171, pp. 1533-1547, 2016. 


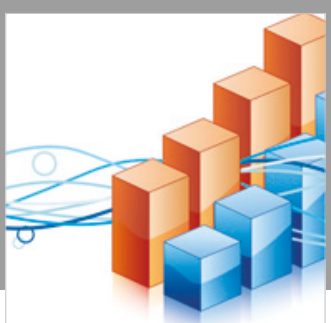

Advances in

Operations Research

\section{-n-m}
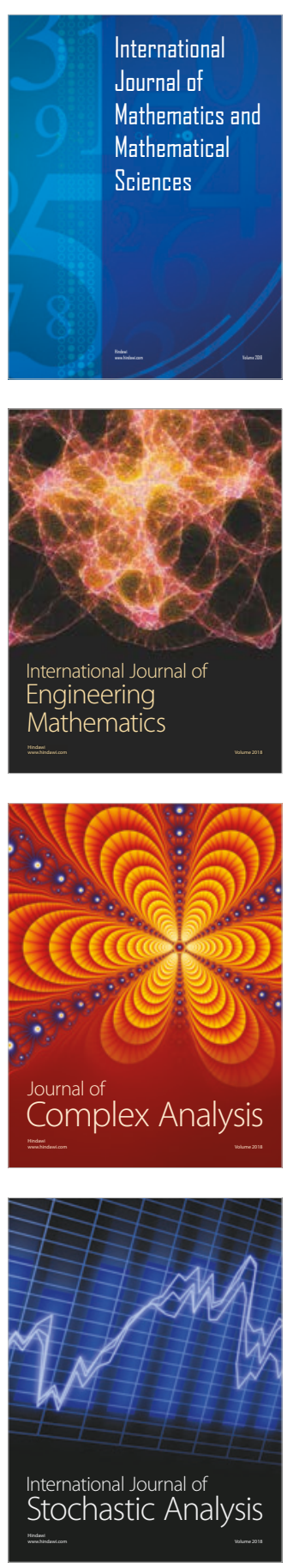
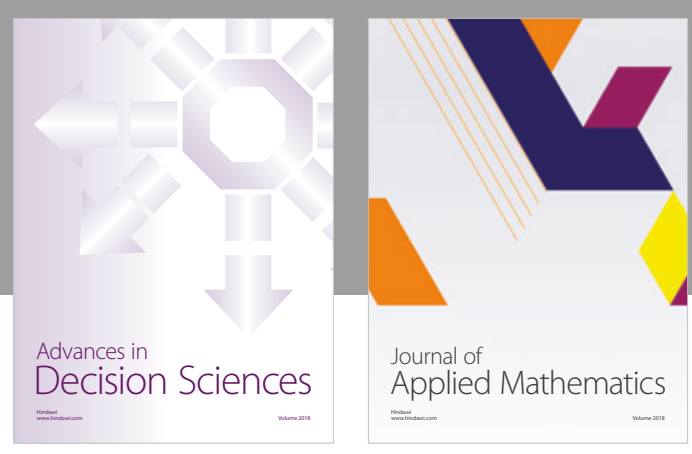

Journal of

Applied Mathematics
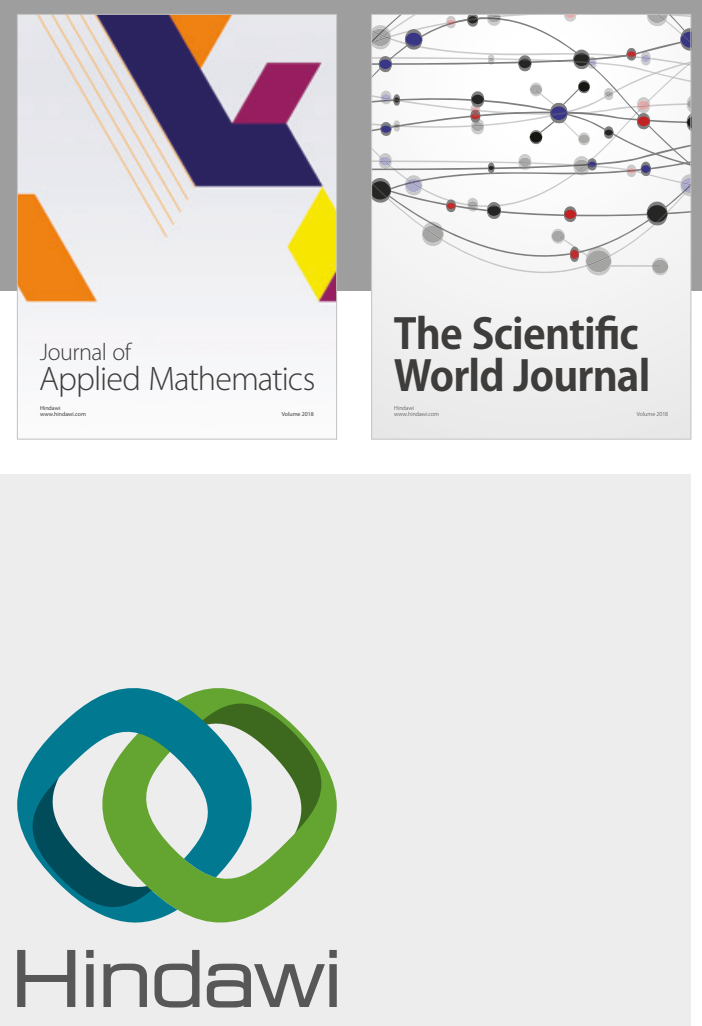

Submit your manuscripts at

www.hindawi.com

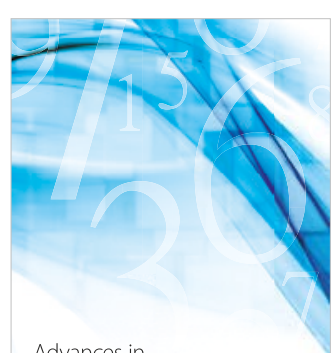

Advances in
Numerical Analysis
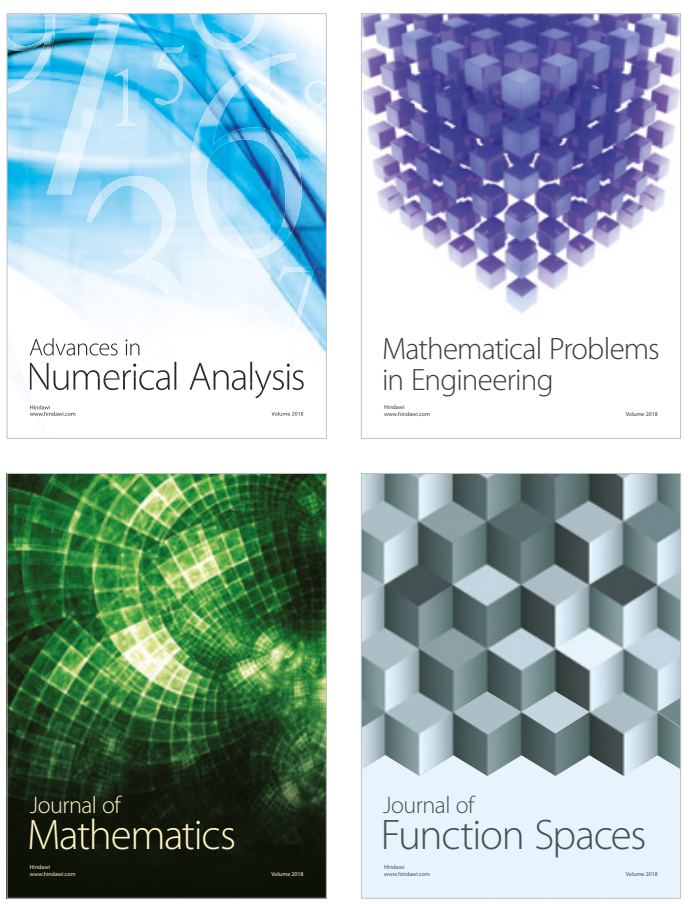

Mathematical Problems in Engineering

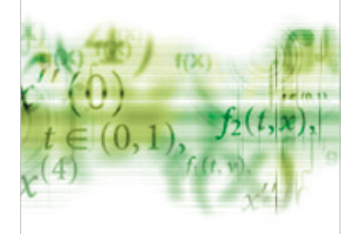

International Journal of

Differential Equations

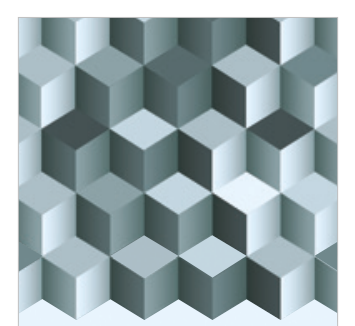

Journal of

Function Spaces

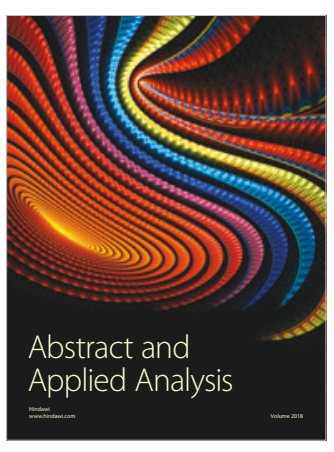

The Scientific

World Journal

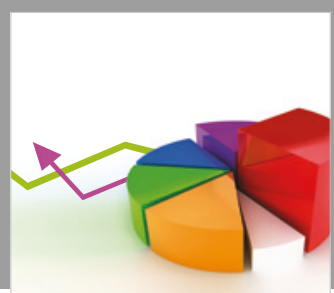

Journal of

Probability and Statistics
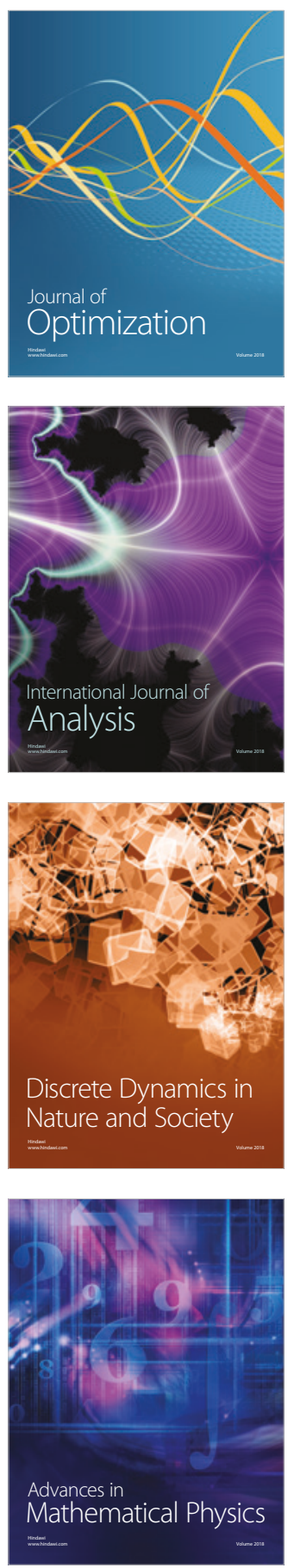